\title{
On the origin of higher braces and higher-order derivations
}

\author{
Martin Markl
}

Received: 13 November 2013 / Accepted: 28 March 2014 / Published online: 1 May 2014

(C) Tbilisi Centre for Mathematical Sciences 2014

\begin{abstract}
The classical Koszul braces, sometimes also called the Koszul hierarchy, were introduced in 1985 by Koszul (Astérisque, (Numero Hors Serie):257-271, 1985). Their non-commutative counterparts came as a surprise much later, in 2013, in a preprint by Börjeson ( $A_{\infty}$-algebras derived from associative algebras with a nonderivation differential, Preprint arXiv:1304.6231, 2013). In Part I we show that both braces are the twistings of the trivial $L_{\infty^{-}}$(resp. $A_{\infty^{-}}$) algebra by a specific automorphism of the underlying coalgebra. This gives an astonishingly simple proof of their properties. Using the twisting, we construct other surprising examples of $A_{\infty}$ and $L_{\infty}$-braces. We finish Part 1 by discussing $C_{\infty}$-braces related to Lie algebras. In Part 2 we prove that in fact all natural braces are the twistings by unique automorphisms. We also show that there is precisely one hierarchy of braces that leads to a sensible notion of higher-order derivations. Thus, the notion of higher-order derivations is independent of human choices. The results of the second part follow from the acyclicity of a certain space of natural operations.
\end{abstract}

Keywords Koszul braces · Börjeson braces $\cdot$ Higher-order derivation

Mathematics Subject Classification (2000) $\quad$ 13D99 · 55S20

Communicated by Jim Stasheff.

The author was supported by the Eduard Čech Institute P201/12/G028 and RVO: 67985840.

M. Markl $(\bowtie)$

Mathematical Institute of the Academy, Žitná 25, 11567 Prague 1, Czech Republic

e-mail: mark1@math.cas.cz

M. Mark1

MFF UK, Sokolovská 83, 18675 Prague 8, Czech Republic 


\section{Contents}

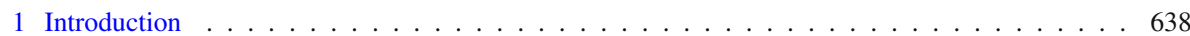

1.1 Examples in place of introduction . . . . . . . . . . . . . . . . . 639

1.2 Higher order derivations . . . . . . . . . . . . . . . . . . . . . . . . . . 640

2 Constructions of higher braces . . . . . . . . . . . . . . . . . . . 643

2.1 Non-commutative algebras and $A_{\infty}$-braces $\ldots \ldots \ldots \ldots \ldots$. . . . . . . . . 643

2.2 Explicit formulas . . . . . . . . . . . . . . . . . . . . . . 645

2.3 Commutative algebras and $L_{\infty}$-braces . . . . . . . . . . . . . . . . . . . . 647

2.4 Explicit formulas . . . . . . . . . . . . . . . . . . . . . . . . . . . . . 648

2.5 The Lie case . . . . . . . . . . . . . . . . . . . . . . . . . . . . . . . . . . . . . . . . . . . . 649

2.6 Other cases . . . . . . . . . . . . . . . . . . . . . . . . . . . . 651

3 Naturality . . . . . . . . . . . . . . . . . . . . . . . . 652

3.1 Natural operations . . . . . . . . . . . . . . . . . . . . . . 652

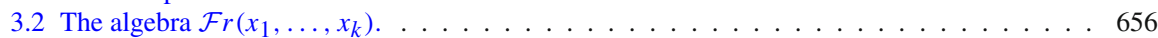

3.3 Acyclicity . . . . . . . . . . . . . . . . . . . . . . . 657

3.4 Natural $A_{\infty}$-algebras . . . . . . . . . . . . . . . . . . . . . . . . . . . . . . . . . . 659

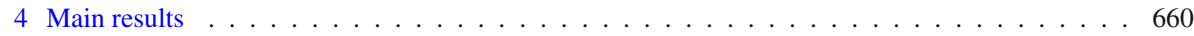

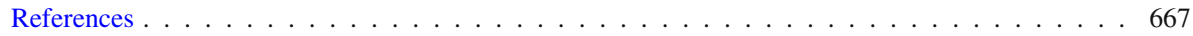

\section{Introduction}

Advice for the reader. The article can be read in four possible ways:

(1) as a tool for checking that the classical braces indeed form $L_{\infty}$-resp. $A_{\infty}$-algebras,

(2) as a machine producing explicit examples of $L_{\infty^{-}}, A_{\infty^{-}}$and possibly also other types of strongly homotopy algebras,

(3) as a justification that the higher-order derivations are God-given, not human, inventions existing since the beginning of time, or

(4) as a vanilla version of [4].

The reader wanting only (1) and (2) may read Part 1 and skip the rest. Item (3) explains why higher-order derivations of commutative associative algebras appear e.g. in the interpretation of the algebraic structure of the combined conformal field theory of matter and ghosts given in [11]. It is natural to expect that higher-order derivations of associative algebras based on Börjeson's braces would play a similar rôle for open strings.

Plan of the paper. Section 1 contains several examples of braces, including the classical Koszul $L_{\infty}$-hierarchy and Börjeson's $A_{\infty}$-braces. We demonstrate various properties which the braces may posses, in particular those leading to a sensible definition of higher-order derivations.

In Sect. 2 we show how to generate braces by the twisting and interpret all examples in Sect. 1 as emerging this way. This offers a very simple verification that they indeed form $L_{\infty}$ - resp. $A_{\infty}$-structures. We close this section by discussing possible generalizations to Lie and other types of algebras.

In Sect. 3 we analyze natural operations and prove that they form an acyclic space. The main results are Propositions 3.3 and 3.6. The material of this section is a baby version of the analysis of the Hochschild cochains in connection to Deligne's conjecture as given in [4]. 
Section 4 formulates the consequences of Sect. 3. Theorems 4.1 and 4.9 state that all natural braces are the twistings by unique automorphisms, Corollaries 4.2 and 4.10 then describe the moduli space of all natural braces. By Theorems 4.3 and 4.11, Börjeson's resp. Koszul braces are the unique ones leading to a meaningful notion of higher-order derivations of associative resp. commutative associative algebras.

Conventions. If not stated otherwise, all algebraic objects will be considered over a fixed field $\mathrm{k}$ of characteristic zero. The symbol $\otimes$ will denote the tensor product over $\mathbb{k}$ and $\operatorname{Span}(S)$ the $\mathrm{k}$-vector space spanned by a set $S$. We will denote by $\mathbb{1}_{X}$ or simply by 11 when $X$ is understood, the identity endomorphism of an object $X$ (set, vector space, algebra, \&c.). We will usually write the product of elements $a$ and $b$ of an associative algebra as $a \cdot b$ or simply as $a b$.

A degree of a graded object will be denoted by $|w|$ though we will sometimes omit the vertical bars and write e.g. $(-1)^{a+b}$ instead of $(-1)^{|a|+|b|}$ to save the space. For a permutation $\sigma \in \Sigma_{k}$ and graded variables $w_{1}, \ldots, w_{k}$, the Koszul sign $\varepsilon\left(\sigma ; w_{1}, \ldots, w_{k}\right) \in\{-1,+1\}$ is defined by the equation

$$
w_{1} \wedge \ldots \wedge w_{k}=\varepsilon\left(\sigma ; w_{1}, \ldots, w_{k}\right) \cdot w_{\sigma(1)} \wedge \ldots \wedge w_{\sigma(k)}
$$

in the free graded commutative associative algebra $\mathbb{S}\left(w_{1}, \ldots, w_{k}\right)$ generated by $w_{1}, \ldots, w_{k}$. We usually write $\varepsilon(\sigma)$ instead of $\varepsilon\left(\sigma ; w_{1}, \ldots, w_{k}\right)$ when the meaning of $w_{1}, \ldots, w_{k}$ is clear from the context.

Given integers $a, b \geq 0$, an $(a, b)$-unshuffle is a permutation $\sigma \in \Sigma_{a+b}$ satisfying

$$
\sigma(1)<\cdots<\sigma(a) \text { and } \sigma(a+1)<\cdots<\sigma(a+b) \text {. }
$$

By braces we mean the structure operations of a strongly homotopy algebra.

\section{Part 1. Examples and constructions}

\subsection{Examples in place of introduction}

We start by recalling a construction attributed to Koszul [7] and sometimes referred to as the Koszul hierarchy, see also [1-3,5, 17]. It is used to define higher order derivations of commutative associative algebras, see $\$ 1.2$ below; they play a substantial rôle for instance in the BRST approach to closed string field theory [11, Section 4].

Example 1.1 (Classical $L_{\infty}$-braces) Let $A$ be a graded commutative associative algebra with a degree +1 differential $\Delta$ which is, very crucially, not necessarily a derivation. Koszul braces are linear degree +1 maps $\Phi_{k}^{\Delta}: A^{\otimes k} \rightarrow A, k \geq 1$, defined by the formulas

$$
\begin{aligned}
\Phi_{1}^{\Delta}(a)= & \Delta(a) \\
\Phi_{2}^{\Delta}\left(a_{1}, a_{2}\right)= & \Delta\left(a_{1} a_{2}\right)-\Delta\left(a_{1}\right) a_{2}-(-1)^{a_{1} a_{2}} \Delta\left(a_{2}\right) a_{1}, \\
\Phi_{3}^{\Delta}\left(a_{1}, a_{2}, a_{3}\right)= & \Delta\left(a_{1} a_{2} a_{3}\right) \\
& -\Delta\left(a_{1} a_{2}\right) a_{3}-(-1)^{a_{1}\left(a_{2}+a_{3}\right)} \Delta\left(a_{2} a_{3}\right) a_{1}-(-1)^{a_{3}\left(a_{1}+a_{2}\right)} \Delta\left(a_{3} a_{1}\right) a_{2}
\end{aligned}
$$




$$
\begin{gathered}
\quad+\Delta\left(a_{1}\right) a_{2} a_{3}+(-1)^{a_{1}\left(a_{2}+a_{3}\right)} \Delta\left(a_{2}\right) a_{3} a_{1}+(-1)^{a_{3}\left(a_{1}+a_{2}\right)} \Delta\left(a_{3}\right) a_{1} a_{2}, \\
\vdots \\
\Phi_{k}^{\Delta}\left(a_{1}, \ldots, a_{k}\right)=\sum_{1 \leq i \leq k}(-1)^{k-i} \sum_{\sigma} \varepsilon(\sigma) \Delta\left(a_{\sigma(1)} \cdots a_{\sigma(i)}\right) a_{\sigma(i+1)} \cdots a_{\sigma(k)},
\end{gathered}
$$

for $a, a_{1}, a_{2}, a_{3}, \ldots \in A$. The summation in the last line runs over all $(i, k-i)$ unshuffles $\sigma$ and $\varepsilon(\sigma)=\varepsilon\left(\sigma ; a_{1}, \ldots, a_{k}\right)$ is the Koszul sign. As proved for instance in [3], these braces form an $L_{\infty}$-algebra ${ }^{1}$ and have moreover the property that

if $\Phi_{k}^{\Delta}=0$ identically on $A^{\otimes k}$ then $\Phi_{k+1}^{\Delta}=0$ identically on $A^{\otimes k+1}$.

We call braces with this property hereditary.

\subsection{Higher order derivations}

Let $A$ be a graded commutative associative algebra with a differential $\Delta$ as in Example 1.1. One says [2] that $\Delta$ is an order $r$ derivation if $\Phi_{r+1}^{\Delta}=0$. Clearly, being an order 1 derivation is the same as being a derivation in the usual sense. It is almost clear that an order $r$-derivation is determined by its values on the products $x_{1} \ldots x_{s}, s \leq r$, of generators of $A$; an explicit formula is given in [11, Proposition 3.4].

One may ask whether higher-order derivations are 'God-given,' i.e. whether the braces that define it are unique. Let us try to find out which properties the braces leading to a sensible notion of higher-order derivations should satisfy. First of all, they must be 'natural' in that they use only the data that are available for any graded associative commutative algebra with a differential. The exact meaning of naturality is analyzed in Sect. 3.

Given $L_{\infty}$-braces $\left(A, \Delta, l_{2}^{\Delta}, l_{3}^{\Delta}, \ldots\right)$, we may call $\Delta$ an order $r l$-derivation if and only if $l_{r+1}^{\Delta}=0$. It is clear from the axioms for $L_{\infty}$-algebras recalled in $\S 2.3$ that, for arbitrary scalars $\alpha, \beta \in \mathbb{k}$, the object

$$
\left(A, \alpha \Delta, \alpha \beta l_{2}^{\Delta}, \alpha \beta^{2} l_{3}^{\Delta}, \alpha \beta^{3} l_{4}^{\Delta}, \ldots\right)
$$

is an $L_{\infty}$-algebra as well. The first property we want is that an order $1 l$-derivation is an ordinary derivation. This means that, after a suitable renormalization (2),

$$
l_{2}^{\Delta}\left(a_{1}, a_{2}\right)=\Delta\left(a_{1} a_{2}\right)-\Delta\left(a_{1}\right) a_{2}-(-1)^{\left|a_{1}\right|} a_{1} \Delta\left(a_{2}\right), \text { for each } a_{1}, a_{2} \in A .
$$

We also certainly want that an order $r l$-derivation is also an order $r+1 l$-derivation, that is:

$$
\text { the braces } l_{2}^{\Delta}, l_{3}^{\Delta}, l_{4}^{\Delta}, \ldots \text { are hereditary. }
$$

The following example however shows that conditions (3)-(4) still do not determine the braces uniquely.

\footnotetext{
${ }^{1}$ We will give a short and elegant proof of this fact in Example $2.8 ; L_{\infty}$-algebras are recalled in Definition 2.6.
} 
Example 1.2 (Hereditary exotic $L_{\infty}$-braces) Here $A$ is a graded commutative associative algebra with a differential $\Delta$ of degree +1 as in Example 1.1. For $a, a_{1}, a_{2}, a_{3}, \ldots \in A$ define

$$
\begin{aligned}
h_{1}^{\Delta}(a)= & \Delta(a), \\
h_{2}^{\Delta}\left(a_{1}, a_{2}\right)= & \Delta\left(a_{1} a_{2}\right)-\Delta\left(a_{1}\right) a_{2}-(-1)^{a_{1} a_{2}} \Delta\left(a_{2}\right) a_{1}, \\
h_{3}^{\Delta}\left(a_{1}, a_{2}, a_{3}\right)= & 2 \Delta\left(a_{1}\right) a_{2} a_{3}+(-1)^{a_{1}\left(a_{2}+a_{3}\right)} 2 \Delta\left(a_{2}\right) a_{3} a_{1}+(-1)^{a_{3}\left(a_{1}+a_{2}\right)} 2 \Delta\left(a_{3}\right) a_{1} a_{2} \\
& -\Delta\left(a_{1} a_{2}\right) a_{3}-(-1)^{a_{1}\left(a_{2}+a_{3}\right)} \Delta\left(a_{2} a_{3}\right) a_{1}-(-1)^{a_{3}\left(a_{1}+a_{2}\right)} \Delta\left(a_{3} a_{1}\right) a_{2}, \\
\vdots & \\
h_{k}^{\Delta}\left(a_{1}, \ldots, a_{k}\right)= & (-1)^{k+1}(k-1) ! \sum_{\tau} \varepsilon(\tau) \Delta\left(a_{\tau(1)}\right) a_{\tau(2)} \cdots a_{\tau(k)} \\
& +(-1)^{k}(k-2) ! \sum_{\sigma} \varepsilon(\sigma) \Delta\left(a_{\sigma(1)} a_{\sigma(2)}\right) a_{\sigma(3)} \cdots a_{\sigma(k)}
\end{aligned}
$$

where $\tau$ runs over all $(1, k-1)$-unshuffles and $\sigma$ over all $(2, k-2)$-unshuffles. It is easy to verify that the above braces satisfy the induction

$$
h_{k+1}^{\Delta}\left(a_{1}, \ldots, a_{k+1}\right)=-\sum_{\sigma} \varepsilon(\sigma) h_{k}^{\Delta}\left(a_{\sigma(1)}, \ldots, a_{\sigma(k)}\right) a_{\sigma(k+1)}
$$

with $\sigma$ running over all $(k, 1)$-unshuffles. This implies that they are hereditary.

Next, we want the recursivity of higher-order derivations, by which we mean that an order $r l$-derivation is determined by its values on the products of $\leq r$ generators. Moreover, the notion of higher-order derivations and therefore the braces as well must be defined over an arbitrary ring. The recursivity is thus equivalent to:

The braces are defined over the ring $\mathbb{Z}$ of integers and the coefficient $C_{k}$ at the term $\Delta\left(a_{1} \cdots a_{k}\right)$ in $l_{k}^{\Delta}\left(a_{1}, \ldots, a_{k}\right)$ is either +1 or -1 for any $k \geq 1$.

For, if $p:=C_{k} \notin\{-1,1\}$ for some $k$, then higher-order $l$-derivations will not be recursive over the ring $\mathbb{Z} / p \mathbb{Z}$ of integers modulo $p$. For the braces in Example 1.2, $C_{k}=0$ for all $k \geq 3$, so they do not satisfy (5).

It will follow from Theorem 4.11 that assumptions (3)-(5) already imply that $l_{k}^{\Delta}=$ $\Phi_{k}^{\Delta}$ for each $k \geq 1$. Let us start our discussion of the non-commutative case by recalling one construction from a recent preprint [6] of Börjeson.

Example 1.3 (Börjeson's $A_{\infty}$-braces) Given a graded associative (not necessarily commutative) algebra $A$ with a differential $\Delta$, define for $a, a_{1}, a_{2}, a_{3}, \ldots \in A$, 


$$
\begin{aligned}
b_{1}^{\Delta}(a)= & \Delta(a), \\
b_{2}^{\Delta}\left(a_{1}, a_{2}\right)= & \Delta\left(a_{1} a_{2}\right)-\Delta\left(a_{1}\right) a_{2}-(-1)^{a_{1}} a_{1} \Delta\left(a_{2}\right), \\
b_{3}^{\Delta}\left(a_{1}, a_{2}, a_{3}\right)= & \Delta\left(a_{1} a_{2} a_{3}\right)-\Delta\left(a_{1} a_{2}\right) a_{3}-(-1)^{a_{1}} a_{1} \Delta\left(a_{2} a_{3}\right)+(-1)^{a_{1}} a_{1} \Delta\left(a_{2}\right) a_{3}, \\
b_{4}^{\Delta}\left(a_{1}, a_{2}, a_{3}, a_{4}\right)= & \Delta\left(a_{1} a_{2} a_{3} a_{4}\right) \\
& -\Delta\left(a_{1} a_{2} a_{3}\right) a_{4}-(-1)^{a_{1}} a_{1} \Delta\left(a_{2} a_{3} a_{4}\right)+(-1)^{a_{1}} a_{1} \Delta\left(a_{2} a_{3}\right) a_{4}, \\
\vdots & \\
b_{k}^{\Delta}\left(a_{1}, \ldots, a_{k}\right)= & \Delta\left(a_{1} \cdots a_{k}\right)-\Delta\left(a_{1} \cdots a_{k-1}\right) a_{k} \\
& -(-1)^{a_{1}} a_{1} \Delta\left(a_{2} \cdots a_{k}\right)+(-1)^{a_{1}} a_{1} \Delta\left(a_{2} \cdots a_{k-1}\right) a_{k} .
\end{aligned}
$$

As proved in [6], these braces form an $A_{\infty}$-algebra ${ }^{2}$ and are hereditary.

It is obvious that Börjeson's braces satisfy assumptions (3)-(5), so they lead to a sensible notion of higher-order derivations of graded associative (non-commutative) algebras. By Theorem 4.3, they are the only $A_{\infty}$-braces with these properties.

Example 1.4 (Non-recursive $A_{\infty}$-braces) The braces below lead to recursive higherorder derivations over $\mathbb{Z}$ but not over $\mathbb{Z} / 5 \mathbb{Z}$, the integers modulo 5 . They are, up to the obvious Koszul signs, given by

$$
\begin{aligned}
e_{1}^{\Delta}(a)= & \Delta(a), \\
e_{2}^{\Delta}\left(a_{1}, a_{2}\right)= & \Delta\left(a_{1} a_{2}\right)-\Delta\left(a_{1}\right) a_{2}-a_{1} \Delta\left(a_{2}\right) \\
e_{3}^{\Delta}\left(a_{1}, a_{2}, a_{3}\right)= & 2 \Delta\left(a_{1} a_{2} a_{3}\right)-\Delta\left(a_{1}\right) a_{2} a_{3}-a_{1} a_{2} \Delta\left(a_{3}\right)-\Delta\left(a_{1} a_{2}\right) a_{3}-a_{1} \Delta\left(a_{2} a_{3}\right), \\
e_{4}^{\Delta}\left(a_{1}, a_{2}, a_{3}, a_{4}\right)= & 5 \Delta\left(a_{1} a_{2} a_{3} a_{4}\right)-\Delta\left(a_{1} a_{2}\right) a_{3} a_{4}-a_{1} a_{2} \Delta\left(a_{3} a_{4}\right) \\
& -2\left(\Delta\left(a_{1}\right) a_{2} a_{3} a_{4}+a_{1} a_{2} a_{3} \Delta\left(a_{4}\right)+\Delta\left(a_{1} a_{2} a_{3}\right) a_{4}+a_{1} \Delta\left(a_{2} a_{3} a_{4}\right)\right) \\
\vdots & \\
e_{k}^{\Delta}\left(a_{1}, \ldots, a_{k}\right)= & \alpha_{k} \Delta\left(a_{1} \cdots a_{k}\right) \\
& -\sum_{1 \leq u \leq k-1} \alpha_{u} \alpha_{k-u}\left(\Delta\left(a_{1} \cdots a_{u}\right) a_{u+1} \cdots a_{k}\right. \\
& \left.+a_{1} \cdots a_{u} \Delta\left(a_{u+1} \cdots a_{k}\right)\right),
\end{aligned}
$$

where

$$
\alpha_{1}:=1 \text { and } \alpha_{k}:=\frac{1}{k-1}\left(\begin{array}{c}
2 k-2 \\
k
\end{array}\right) \text { for } k \geq 2 \text {. }
$$

\footnotetext{
2 A simple proof of this fact is provided by Example 2.4; $A_{\infty}$-algebras are recalled in Definition 2.1.
} 
Example 1.5 (Hereditary non-recursive $A_{\infty}$-braces) We define braces satisfying (3), (4) but not (5). Namely, for elements $a, a_{1}, a_{2}, a_{3}, \ldots$ of a graded associative algebra $A$ with a differential $\Delta$ we put

$$
\begin{aligned}
e_{1}^{\Delta}(a)= & \Delta(a), \\
e_{2}^{\Delta}\left(a_{1}, a_{2}\right)= & \Delta\left(a_{1} a_{2}\right)-\Delta\left(a_{1}\right) a_{2}-a_{1} \Delta\left(a_{2}\right), \\
e_{3}^{\Delta}\left(a_{1}, a_{2}, a_{3}\right)= & -\Delta\left(a_{1} a_{2}\right) a_{3}-a_{1} \Delta\left(a_{2} a_{3}\right)+\Delta\left(a_{1}\right) a_{2} a_{3}+2 a_{1} \Delta\left(a_{2}\right) a_{3}+a_{1} a_{2} \Delta\left(a_{3}\right), \\
e_{4}^{\Delta}\left(a_{1}, a_{2}, a_{3}, a_{4}\right)= & \Delta\left(a_{1} a_{2}\right) a_{3} a_{4}+2 a_{1} \Delta\left(a_{2} a_{3}\right) a_{4}+a_{1} a_{2} \Delta\left(a_{3} a_{4}\right) \\
& -\Delta\left(a_{1}\right) a_{2} a_{3} a_{4}-3 a_{1} \Delta\left(a_{2}\right) a_{3} a_{4}-3 a_{1} a_{2} \Delta\left(a_{3}\right) a_{4}-a_{1} a_{2} a_{3} \Delta\left(a_{4}\right), \\
\vdots & \\
e_{k}^{\Delta}\left(a_{1}, \ldots, a_{k}\right)= & (-1)^{k} \sum_{0 \leq i \leq k-2}\left(\begin{array}{c}
k-2 \\
i
\end{array}\right) a_{1} \cdots \Delta\left(a_{i+1} a_{i+2}\right) \cdots a_{k} \\
& -(-1)^{k} \sum_{0 \leq i \leq k-1}\left(\begin{array}{c}
k-1 \\
i
\end{array}\right) a_{1} \cdots \Delta\left(a_{i+1}\right) \cdots a_{k} .
\end{aligned}
$$

We omitted for clarity the obvious Koszul signs. It is easy to verify the inductive formula

$e_{k+1}^{\Delta}\left(a_{1}, \ldots, a_{k+1}\right)=-(-1)^{a_{1}} a_{1} e_{k}^{\Delta}\left(a_{2}, \ldots, a_{k+1}\right)-e_{k}^{\Delta}\left(a_{1}, \ldots, a_{k}\right) a_{k+1}, k \geq 1$,

which implies that they are hereditary. On the other hand, $e_{k}^{\Delta}\left(a_{1}, \ldots, a_{k}\right)$ does not contain the term $\Delta\left(a_{1} \cdots a_{k}\right)$, so the coefficients $C_{k}$ in (5) are 0 for all $k \geq 2$.

Hereditarity is a very fine property; 'randomly chosen' braces will not be hereditary. A systematic method of producing non-hereditary braces, based surprisingly on a rather deep Proposition 4.4, is described in Example 4.7.

\section{Constructions of higher braces}

2.1 Non-commutative algebras and $A_{\infty}$-braces

Recall that an $A_{\infty}$-algebra consists of a graded vector space $V$ together with linear operations $\mu_{k}: V^{\otimes k} \rightarrow V, k \geq 1$, such that $\operatorname{deg}\left(\mu_{k}\right)=2-k$, satisfying a system of axioms that say that $\mu_{1}$ is a differential, $\mu_{2}$ is associative up to the homotopy $\mu_{3}, \& \mathrm{c}$, see e.g. $[15,16]$.

It will be useful in the context of this paper to transfer the operations $\mu_{k}: V^{\otimes k} \rightarrow V$ to the desuspension $A:=\downarrow V$, i.e. to define new operations $m_{k}: A^{\otimes k} \rightarrow A$ by the commutativity of the diagram

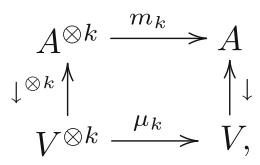


where $\downarrow: V \rightarrow \downarrow V=A$ is the desuspension map. All $m_{k}$ 's then are of degree +1 and they satisfy the axioms

$$
\sum_{k+l=n+1} \sum_{1 \leq i \leq k} m_{k}\left(\mathbb{1}_{A}^{\otimes i-1} \otimes m_{l} \otimes \mathbb{1}_{A}^{\otimes k-i}\right)=0, \text { for each } n \geq 1 .
$$

We will use this version of $A_{\infty}$-algebras throughout the paper:

Definition 2.1 An $A_{\infty}$-algebra is a structure $\mathcal{A}=\left(A, m_{1}, m_{2}, m_{3}, \ldots\right)$ consisting of a graded vector space $A$ and degree +1 linear maps $m_{k}: A^{\otimes k} \rightarrow A, k \geq 1$, satisfying (7).

Let $\mathbb{T}^{c} A$ be the coalgebra whose underlying space is the tensor algebra $\mathbb{T} A:=$ $\bigoplus_{n>1} A^{\otimes n}$ and the diagonal (comultiplication) is the de-concatenation. It turns out that $\mathbb{T}^{c} A$ is a cofree conilpotent coassociative coalgebra cogenerated by $A$, see e.g. [13, $\S I I .3 .7] .^{3}$ Its cofreeness implies that each coderivation $\vartheta$ of $\mathbb{T}^{c} A$ is given by its components $\vartheta_{k}: A^{\otimes k} \rightarrow A, k \geq 1$, defined by $\vartheta_{k}:=\pi \circ \vartheta \circ \iota_{k}$, where $\pi: \mathbb{T}^{c} A \rightarrow A$ is the projection and $\iota_{k}: A^{\otimes k} \hookrightarrow \mathbb{T}^{c} A$ the inclusion. We write $\vartheta=\left(\vartheta_{1}, \vartheta_{2}, \vartheta_{3}, \ldots\right)$.

Let $m:=\left(m_{1}, m_{2}, m_{3}, \ldots\right)$ be a degree 1 coderivation of $\mathbb{T}^{c} A$ determined by the linear maps $m_{k}$ as in Definition 2.1. It is well-known that axiom (7) is equivalent to $m$ being a differential, i.e. to a single equation $m^{2}=0$. Therefore equivalently, an $A_{\infty}$-algebra is a pair $(A, m)$ consisting of a graded vector space $A$ and a degree +1 coderivation $m$ of $\mathbb{T}^{c} A$ which squares to zero.

In homological algebra one usually considers $A_{\infty}$-algebras $\left(A, m_{1}, m_{2}, m_{3}, \ldots\right)$ as objects living in the category of differential graded $(\mathrm{dg})$ vector spaces, the linear operation (differential) $m_{1}$ being part of its underlying dg-vector space, not a structure operation. For this reason we call an $A_{\infty}$-algebra with $m_{k}=0$ for $k \geq 2$ a trivial $A_{\infty}$-algebra.

Example 2.2 (Trivial $A_{\infty}$-algebra) Let $\Delta: A \rightarrow A$ be a degree +1 differential on a graded vector space $A$. It is clear that $\mathcal{A}_{\Delta}:=(A, \Delta, 0,0, \ldots)$ is an $A_{\infty}$-algebra. The differential $\Delta$ extends to a linear coderivation $(\Delta, 0,0, \ldots)$ of $\mathbb{T}^{c} A$.

As coderivations, by the universal property of $\mathbb{T}^{c} A$ each endomorphism $\phi: \mathbb{T}^{c} A \rightarrow$ $\mathbb{T}^{c} A$ is determined by its components $\phi_{k}: A^{\otimes k} \rightarrow A, k \geq 1$, defined by $\phi_{k}:=$ $\pi \circ \phi \circ \iota_{n}$, We will write $\phi=\left(\phi_{1}, \phi_{2}, \phi_{3}, \ldots\right)$. The sequence $\left(\mathbb{1}_{A}, 0,0, \ldots\right)$ represents the identity automorphism. The composition $\psi \phi$ of $\phi$ with another endomorphism $\psi=\left(\psi_{1}, \psi_{2}, \psi_{3}, \ldots\right)$ has components

$$
(\psi \phi)_{k}=\sum_{r \geq 1} \sum_{i_{1}+\cdots+i_{r}=k} \psi_{r}\left(\phi_{i_{1}} \otimes \cdots \otimes \phi_{i_{r}}\right) .
$$

It is well-known that $\phi: \mathbb{T}^{c} A \rightarrow \mathbb{T}^{c} A$ is an automorphism (i.e. invertible endomorphism) if and only if $\phi_{1}: A \rightarrow A$ is invertible. We call $\phi$ linear if $\phi_{k}=0$ for $k \geq 2$. Let us recall

\footnotetext{
3 A general misconception is that $\mathbb{T}^{c} A$ is cofree in the category of all coassociative coalgebras.
} 
Definition Two $A_{\infty}$-algebras $\mathcal{A}^{\prime}=\left(A^{\prime}, m^{\prime}\right)$ and $\mathcal{A}^{\prime \prime}=\left(A^{\prime}, m^{\prime \prime}\right)$ are isomorphic if there exists an automorphism $\phi: \mathbb{T}^{c} A^{\prime} \rightarrow \mathbb{T}^{c} A^{\prime \prime}$ such that $\phi m^{\prime}=m^{\prime \prime} \phi{ }^{4}$ They are strictly isomorphic if there exist a linear $\phi$ as above.

Assume that we are given an $A_{\infty}$-algebra $\mathcal{A}=(A, m)$ and an automorphism $\phi: \mathbb{T}^{c} A \rightarrow \mathbb{T}^{c} A$. Then clearly $\left(A, \phi^{-1} m \phi\right)$ is an $A_{\infty}$-algebra isomorphic to $\mathcal{A}$.

Definition 2.3 In the situation above, we denote $m^{\phi}:=\phi^{-1} m \phi$ and call the $A_{\infty^{-}}$ algebra $\mathcal{A}^{\phi}:=\left(A, m^{\phi}\right)$ the twisting of the $A_{\infty}$-algebra $\mathcal{A}=(A, m)$ by the automorphism $\phi$.

The components of the twisted coderivation $m^{\phi}:=\phi^{-1} m \phi$ can be expressed explicitly as

$m_{k}^{\phi}=\sum_{r, u \geq 1} \sum_{1 \leq j \leq r} \sum_{i_{1}+\cdots+i_{r+u-1}=k}\left(\phi^{-1}\right)_{r}\left(\mathbb{1}^{\otimes j-1} \otimes m_{u} \otimes \mathbb{1}^{\otimes r-j}\right)\left(\phi_{i_{1}} \otimes \cdots \otimes \phi_{i_{r+u-1}}\right)$.

If $m$ is the linear coderivation $\Delta$ as in Example 2.2, the above formula simplifies to

$$
\Delta_{k}^{\phi}=\sum_{r \geq 1} \sum_{1 \leq j \leq r} \sum_{i_{1}+\cdots+i_{r}=k}\left(\phi^{-1}\right)_{r}\left(\mathbb{1}^{\otimes j-1} \otimes \Delta \otimes \mathbb{1}^{\otimes r-j}\right)\left(\phi_{i_{1}} \otimes \cdots \otimes \phi_{i_{r}}\right) .
$$

\subsection{Explicit formulas}

Let $A$ be graded associative algebra with the product $\mu: A^{\otimes 2} \rightarrow A$. Denote by $\underline{\operatorname{Aut}}(A)$ the group of automorphisms $\phi$ of $\mathbb{T}^{c}(A)$ of the form

$$
\phi:=\left(\mathbb{1}_{A}, f_{2} \mu_{2}, f_{3} \mu_{3}, f_{4} \mu_{4}, \ldots\right),
$$

where $\mu_{k}: A^{\otimes k} \rightarrow A$ is the multiplication $\mu$ iterated $(k-1)$-times, and $f_{k} \in \mathbb{k}$ are scalars, $k \geq 2 .^{5}$ Such an automorphism is clearly determined by its generating series

$$
\phi(t):=t+f_{2} t^{2}+f_{3} t^{3}+\cdots \in \mathbb{k}[[t]]
$$

It is easy to verify using (8) that the composition of automorphisms is translated into the composition of their generating series, i.e. $(\psi \phi)(t)=\psi(\phi(t))$.

Let $\phi \in \underline{\operatorname{Aut}}(A)$ be the automorphism with the generating series (10) and $\psi(t):=$ $\phi^{-1}(t)$ its inverse with the generating series

$$
\psi(t)=t+g_{2} t^{2}+g_{3} t^{3}+\cdots \in \mathbb{k}[[t]]
$$

Denote by

$$
\psi^{\prime}(t):=1+g_{2} t+g_{3} t^{2}+g_{4} t^{3} \cdots \in \mathbb{R}[[t]]
$$

\footnotetext{
4 Sometimes one says that $\mathcal{A}^{\prime}$ and $\mathcal{A}^{\prime \prime}$ are weakly isomorphic.

5 As explained in Example 3.8, $\underline{\operatorname{Aut}}(A)$ is a sub-monoid of the monoid of all natural automorphisms.
} 
the noncommutative derivative of $\psi(t)$. It is straightforward to verify that (9) gives

$$
\Delta_{k}^{\phi}=\sum_{r+s+p=k} c_{r, s} \cdot f_{p} \cdot \mu_{r+s+1}\left(\mathbb{1}^{\otimes r} \otimes \Delta \mu_{p} \otimes \mathbb{1}^{\otimes s}\right),
$$

with the coefficients $c_{r, s} \in \mathbb{k}$ defined as

$$
c_{r, s}:=\left.\psi^{\prime}(\phi(u)+\phi(v))\right|_{u^{r} v^{s}},
$$

where $\left.\right|_{u^{r} v^{s}}$ denotes the coefficient at $u^{r} v^{s}$ of the corresponding power series in the ring of noncommutative polynomials in $u$ and $v$, i.e. the noncommutative Taylor coefficient at $u^{r} v^{s}$. Explicitly,

$$
c_{r, s}=\sum_{k, l \geq 0} g_{k+l+1} \sum_{a_{1}+\cdots+a_{k}=r} f_{a_{1}} \cdots f_{a_{k}} \sum_{b_{1}+\cdots+b_{l}=s} f_{b_{1}} \cdots f_{b_{l}} \text {, }
$$

where we put, by definition, $g_{1}=f_{1}:=1$. Observe that the above sum makes sense even for $r$ or $s$ equaling 0 provided we interpret the empty product as 1 .

Exercise If $\Delta$ is a derivation, then $\Delta^{\phi}=\Delta$, i.e. $\Delta_{k}=0$ in (11) for all $k \geq 2$.

Example 2.4 (Börjeson's $A_{\infty}$-braces continued) We describe the braces constructed in [6] and recalled in Example 1.3 as a twisting of the trivial $A_{\infty}$-algebra $\mathcal{A}_{\Delta}=$ $(A, \Delta, 0,0, \ldots)$. We take as $\phi$ the automorphism with the generating series

$$
\phi(t):=t+t^{2}+t^{3}+\cdots=\frac{t}{1-t},
$$

so that

$$
\psi(t):=\phi^{-1}(t)=t-t^{2}+t^{3}-\cdots=\frac{t}{1+t} .
$$

In this case, $g_{k+l+1}$ in (13) equals $(-1)^{k+l}$, therefore

$$
\begin{aligned}
c_{r, s} & =\sum_{a_{1}+\cdots+a_{k}=r}(-1)^{k} f_{1} \cdots f_{a_{k}} \sum_{b_{1}+\cdots+b_{l}=s}(-1)^{l} f_{b_{1}} \cdots f_{b_{l}} \\
& =\left.(1-\psi \phi(u))(1-\psi \phi(v))\right|_{u^{r} v^{s}}=\left.(1-u)(1-v)\right|_{u^{r} v^{s}} .
\end{aligned}
$$

We conclude that

$$
c_{r, s}= \begin{cases}1 & \text { if }(r, s) \in\{(0,0),(1,1)\}, \\ -1 & \text { if }(r, s) \in\{(0,1),(1,0)\}, \quad \text { and } \\ 0 & \text { in the remaining cases }\end{cases}
$$

Since $f_{p}=1$, formula (11) obviously gives Börjeson' braces, i.e. $b_{k}^{\Delta}=\Delta_{k}^{\phi}$ for each $k \geq 1$. 
Example 2.5 (Non-recursive $A_{\infty}$-braces continued) The braces described in Example 1.4 are the result of the twisting by the endomorphism $\phi$ with the generating function

$$
\phi(t):=\frac{1-\sqrt{1-4 t}}{2}=t+\sum_{k \geq 2} \frac{t^{k}}{k-1}\left(\begin{array}{c}
2 k-2 \\
k
\end{array}\right)=t+t^{2}+2 t^{3}+5 t^{4}+14 t^{5}+\cdots,
$$

whose inverse equals $\psi(t)=t-t^{2}$. Observe that $\psi^{\prime}(t)=1-t$, therefore, in (12)

$c_{r, s}=\left.(1-\phi(u)-\phi(v))\right|_{u^{r} v^{s}}= \begin{cases}-\left(\alpha_{r}+\alpha_{s}\right) & \text { if } r=0 \text { or } s=0 \text { but }(r, s) \neq(0,0), \\ 1 & \text { if }(r, s)=(0,0), \text { and } \\ 0 & \text { in the remaining cases, }\end{cases}$

where $\alpha_{i}$ 's are the Taylor coefficients of $\phi(t)$. It is simple to verify that formula (11) leads to the braces of Example 1.4.

Example (Hereditary non-recursive $A_{\infty}$-braces continued) The braces in Example 1.5 are generated by the automorphism with the generating series $\phi(t)=t+t^{2}$ whose inverse is

$$
\begin{aligned}
\psi(t): & =\frac{\sqrt{1+4 t}-1}{2}=t-\sum_{k \geq 2} \frac{(-t)^{k}}{k-1}\left(\begin{array}{c}
2 k-2 \\
k
\end{array}\right) \\
& =t-t^{2}+2 t^{3}-5 t^{4}+14 t^{5}-\cdots
\end{aligned}
$$

We leave as an exercise to perform the calculation. Notice that $\phi(t)$ and $\psi(t)$ are related with those from Example 1.5 via the transformation

$$
\phi(t) \mapsto-\psi(-t), \psi(t) \mapsto-\phi(-t)
$$

\subsection{Commutative algebras and $L_{\infty}$-braces}

Lie counterparts of $A_{\infty}$-algebras are $L_{\infty}$-algebras. An $L_{\infty}$-algebra is a graded vector space $L$ with linear operations $\ell_{k}: L^{\otimes k} \rightarrow V, k \geq 1, \operatorname{deg}\left(\ell_{k}\right)=2-k$, that are graded antisymmetric and satisfy axioms that say that $\ell_{1}$ is a differential, $\ell_{2}$ fulfills the Jacobi identity up to the homotopy $\ell_{3}$, \&c, see e.g. [8,9].

As for $A_{\infty}$-algebras, we will use the version transferred to the desuspension $A:=$ $\downarrow L$. The transferred structure operations $l_{k}$ 's have degree +1 , are graded symmetric, and satisfy, for each $a_{1}, \ldots, a_{n} \in A$, the 'master identity'

$$
\sum_{i+j=n+1} \sum_{\sigma} \varepsilon(\sigma) l_{j}\left(l_{i}\left(a_{\sigma(1)}, \ldots, a_{\sigma(i)}\right), a_{\sigma(i+1)}, \ldots, a_{\sigma(n)}\right)=0,
$$

where $\sigma$ runs over all $(i, n-i)$-unshuffles and $\varepsilon(\sigma)$ is the Koszul sign of $\sigma$. We thus use: 
Definition 2.6 An $L_{\infty}$-algebra is an object $\mathcal{L}=\left(A, l_{1}, l_{2}, l_{3}, \ldots\right)$ consisting of a graded vector space $A$ and degree +1 graded symmetric linear maps $l_{k}: A^{\otimes k} \rightarrow A$, $k \geq 1$, satisfying (14) for each $n \geq 1$.

Let $\mathbb{S}^{c} A=\bigoplus_{k \geq 1} \mathbb{S}^{k} A$ be the symmetric coalgebra with the diagonal given by the de-concatenation; it is the cofree conilpotent cocommutative coassociative coalgebra cogenerated by $A$. Each coderivation $\omega$ of $\mathbb{S}^{c} A$ is thus determined by its components $\omega_{k}:=\pi \circ \omega \circ \iota_{k}, k \geq 1$, where $\pi: \mathbb{S}^{c} A \rightarrow A$ is the projection and $\iota_{k}: \mathbb{S}^{k} A \hookrightarrow \mathbb{S}^{c} A$ the inclusion of the $k$ th symmetric power of $A$. We write $\omega=\left(\omega_{1}, \omega_{2}, \omega_{3}, \ldots\right)$.

Let $l:=\left(l_{1}, l_{2}, l_{3}, \ldots\right)$ be a degree 1 coderivation of $\mathbb{S}^{c} A$ determined by the linear maps $l_{k}$ of Definition 2.6. Axiom (14) is equivalent to a single equation $l^{2}=0$ [8, Theorem 2.3]. So an $L_{\infty}$-algebra is a pair $(A, l)$ a graded vector space and a degree +1 coderivation $l$ of $\mathbb{S}^{c} A$ which squares to zero.

Example 2.7 (Trivial $L_{\infty}$-algebra) The observations of Example 2.2 apply verbatim to the $L_{\infty}$-case - if $\Delta$ is a degree +1 differential on a graded vector space $A$, then $\mathcal{L}_{\Delta}:=(A, \Delta, 0,0, \ldots)$ is an $L_{\infty}$-algebra.

As automorphisms of $\mathbb{T}^{c} A$ twist $A_{\infty}$-algebras, $L_{\infty}$-algebras can be twisted by automorphisms $\phi: \mathbb{S}^{c} A \rightarrow \mathbb{S}^{c} A$ determined by their components $\phi_{k}: \mathbb{S}^{k} A \rightarrow A$, $k \geq 1$. We leave as an exercise to derive formulas for the composition and for the twisting of $\mathcal{L}_{\Delta}$ analogous to (8) and (9).

\subsection{Explicit formulas}

For a graded associative commutative algebra with a multiplication $\mu: A^{\otimes 2} \rightarrow A$, denote by $\operatorname{Aut}(A)$ the group of automorphisms $\phi$ of $\mathbb{S}^{c}(A)$ of the form

$$
\phi:=\left(\mathbb{1}_{A}, f_{2} \mu_{2}, f_{3} \mu_{3}, f_{4} \mu_{4}, \ldots\right),
$$

where $\mu_{k}: A^{\otimes k} \rightarrow A$ is the multiplication $\mu$ iterated $(k-1)$-times, and $f_{k} \in \mathbb{k}$ are scalars, $k \geq 2$. To such an automorphism we associate its generating series

$$
\phi(t)=1+f_{1} t+\frac{f_{2}}{2 !} t^{2}+\frac{f_{3}}{3 !} t^{3}+\cdots \in \mathbb{k}[[t]] .
$$

It is simple to verify that the generating series of the composition of two automorphisms is the composition of their generating series, i.e. $(\psi \phi)(t)=\psi(\phi(t))$. The situation is analogous to the non-commutative case, only the generating series involve factorials.

Let $\phi \in \operatorname{Aut}(A)$ be the automorphism with the generating series (15) and $\psi(t):=$ $\phi^{-1}(t)$ the inverse of its generating series,

$$
\psi(t)=1+g_{1} t+\frac{g_{2}}{2 !} t^{2}+\frac{g_{3}}{3 !} t^{3}+\cdots \in \mathbb{K}[[t]] .
$$

Denote by

$$
\psi^{\prime}(t):=g_{1}+g_{2} t+\frac{g_{3}}{2 !} t^{2}+\frac{g_{4}}{3 !} t^{3} \cdots \in \mathbb{k}[[t]]
$$


its (ordinary) derivative. It is simple to verify that the components of the twisting $\Delta^{\phi}$ of $\Delta$ via $\phi$ are given by

$$
\begin{aligned}
& \Delta_{k}^{\phi}\left(a_{1}, \ldots, a_{k}\right) \\
& =\sum_{\sigma} \sum_{r+s=k} c_{r} \cdot f_{s} \cdot \varepsilon(\sigma) \mu_{r+1}\left(\Delta \mu_{s}\left(a_{\sigma(1)}, \ldots, a_{\sigma(s)}\right), a_{\sigma(s+1)}, \ldots, a_{\sigma(k)}\right)
\end{aligned}
$$

where $\sigma$ runs over all $(r, s)$-unshuffles, $\varepsilon(\sigma)$ is the Koszul sign of $\sigma$ and

$$
c_{r}:=\left.\frac{d^{r} \psi^{\prime}(\phi(t))}{d t^{r}}\right|_{t=0}
$$

Example 2.8 (Classical $L_{\infty}$-braces) The classical Koszul braces recalled in Example 1.1 are the twisting of $\mathcal{L}_{\Delta}$ by the automorphism with the generating series

$$
\phi(t):=e^{t}-1=\sum_{k \geq 1} \frac{1}{k !} t^{k}=t+\frac{1}{2 !} t^{2}+\frac{1}{3 !} t^{3}+\cdots
$$

whose inverse $\psi(t)$ equals

$$
\psi(t)=\ln (t+1)=\sum_{k \geq 1} \frac{(-1)^{k+1}}{k} t^{k}=t-\frac{t^{2}}{2}+\frac{t^{3}}{3}-\frac{t^{4}}{4}+\cdots
$$

Since $\psi^{\prime}(t)=(1+t)^{-1}, \psi^{\prime}(\phi(t))=e^{-t}$, therefore $c_{r}=(-1)^{r}$. As $f_{s}=1$ for each $s \geq 1$, formula (16) readily gives $\Delta_{k}^{\phi}=\Phi_{k}^{\Delta}$ for each $k \geq 1$.

Example (Hereditary exotic $L_{\infty}$-braces continued) The braces in Example 1.2 are given by the automorphism with the generating series $\phi(t)=t+t^{2} / 2$ whose inverse equals

$$
\begin{aligned}
\psi(t) & =-1+\sqrt{1+2 t}=t-\frac{1}{2} t^{2}+\frac{1}{2} t^{3}-\frac{5}{8} t^{4}+\cdots \\
& =t-\sum_{k \geq 2} \frac{(-t)^{k}}{2^{k-1}(k-1)}\left(\begin{array}{c}
2 k-2 \\
k
\end{array}\right) .
\end{aligned}
$$

Problem It is clear that an automorphism $\phi(t)$ with the generating series (15) leads to recursive braces if and only if $f_{k} \in\{-1,+1\}$. Which property of the generating function guarantees the hereditarity?

\subsection{The Lie case}

One may ask how the previous material translates to the Lie algebra case. One could expect to have, for a graded Lie algebra $L$ with a differential $\Delta$, natural $C_{\infty}$-braces 
$\left(L, \Delta, c_{2}, c_{3}, \ldots\right)$ emerging as the twistings of the trivial $C_{\infty}$-algebra by automorphisms of the Lie coalgebra $\mathbb{L}^{c} L$ and, among these structures, a particular one that leads to higher-order derivations of Lie algebras.

Recall that a $C_{\infty}$-algebra (also called, in [10, $\left.\$ 1.4\right]$, a balanced $A_{\infty}$-algebra) is an $A_{\infty}$-algebra as in Definition 2.1 whose structure operations vanish on decomposables of the shuffle product. As in the $A_{\infty}$ - or $L_{\infty}$-cases, $C_{\infty}$-algebras can equivalently be described as square-zero coderivations of the cofree conilpotent Lie coalgebra $\mathbb{L}^{c} L$ cogenerated by $L$.

We may try to proceed as in the previous two cases. We have the trivial $C_{\infty}$-algebra $\mathcal{C}_{\Delta}=(L, \Delta, 0,0, \ldots)$, thus any natural automorphism $\phi: \mathbb{L}^{c} L \rightarrow \mathbb{L}^{c} L$ determines a coderivation $\Delta^{\phi}:=\phi^{-1} \Delta \phi$ that squares to 0 , i.e. $C_{\infty}$-braces on $L$.

The sting lies in the notion of naturality. In constructing $A_{\infty}$-braces we very crucially relied on the fact that the cofree conilpotent coassociative coalgebra cogenerated by $A$ materialized as the tensor algebra $\mathbb{T} A$ equipped with the de-concatenation diagonal. Therefore natural operations $\mathbb{T} A \rightarrow A$ give rise to natural automorphisms of $\mathbb{T}^{c} A$ and thus also to natural $A_{\infty}$-braces. Similarly, the cofree conilpotent coalgebra cogenerated by $A$ can be realized as the symmetric algebra $\mathbb{S} V$ with the de-concatenation (unshuffle) diagonal.

We were however not able to find an explicit and natural (i.e. not depending e.g. on the choice of a basis) formula for a diagonal on the free Lie algebra $\mathbb{L} L$ that would make it a cofree conilpotent coalgebra; we were able to describe the diagonal for Lie words of length $\leq 3$ only. It is given, for $v, v_{1}, v_{2}, v_{3} \in L$, by

$$
\begin{aligned}
D(v) & =0 \\
D\left\{v_{1}, v_{2}\right\} & =v_{1} \wedge v_{2}, \\
D\left\{v_{1},\left\{v_{2}, v_{3}\right\}\right\} & =2 v_{1} \wedge\left\{v_{2}, v_{3}\right\}-v_{2} \wedge\left\{v_{3}, v_{1}\right\}-v_{3} \wedge\left\{v_{1}, v_{2}\right\} .
\end{aligned}
$$

In the above display, we denoted the bracket in $\mathbb{L} L$ by $\{-,-\}$ to distinguish it from the bracket of $L$ which we will denote more traditionally by $[-,-]$.

Remark 2.9 The lack of an explicit diagonal for the free Lie algebra $\mathbb{L} L$ may be related to the problem of describing the Eulerian idempotents $e_{k}^{(1)}: \mathbb{T}^{k} X \rightarrow \mathbb{T}^{k} X$ [14, Corollary 1.6] in terms of iterated linearly-independent Lie braces. While, for $x, x_{1}, x_{2}, x_{3} \in X$,

$$
\begin{aligned}
e_{1}^{(1)}(x) & =x, \\
e_{2}^{(1)}\left(x_{1} \otimes x_{2}\right) & =\frac{1}{2 !}\left[x_{1}, x_{2}\right] \text { and } \\
e_{2}^{(1)}\left(x_{1} \otimes x_{2} \otimes x_{3}\right) & =\frac{1}{3 !}\left(\left[\left[x_{1}, x_{2}\right], x_{3}\right]+\left[x_{1},\left[x_{2}, x_{3}\right]\right]\right),
\end{aligned}
$$

a similar formula for $e_{k}^{(1)}$ with $k \geq 4$ is not known.

Let $\mathbb{L}^{k} L$ be the subspace of $\mathbb{L} L$ spanned by elements of the product length $k$. As in the previous cases, each coalgebra automorphism $\phi: \mathbb{L}^{c} L \rightarrow \mathbb{L}^{c} L$ is determined by its 
components $\phi_{k}: \mathbb{L}^{k} L \rightarrow L, k \geq 1$. One has also the canonical maps $\lambda_{k}: \mathbb{L}^{k} L \rightarrow L$ given by the multiplication in $L$. Assume we found a natural isomorphism between $\mathbb{L} L$ and $\mathbb{L}^{c} L$ such that the induced diagonal on $\mathbb{L} L$ agrees with (17) on elements of length $\leq 3$.

Let $\phi=\left(\mathbb{1}_{L}, f_{2} \lambda_{2}, f_{3} \lambda_{3}, \ldots\right)$ be the automorphism whose $k$ th component equals $f_{k} \lambda_{k}$ for some scalars $f_{k} \in \mathbb{k}$, and $\psi=\left(\mathbb{1}_{L}, g_{2} \lambda_{2}, g_{3} \lambda_{3}, \ldots\right)$ another one, with components $g_{k} \lambda_{k}, g_{k} \in \mathbb{k}$. Using (17), one derives the following formula for the first three components of the composition:

$$
\psi \phi=\left(\mathbb{1}_{L},\left(f_{2}+g_{2}\right) \lambda_{2},\left(f_{3}+3 f_{2} g_{2}+g_{3}\right) \lambda_{3}, \ldots\right) .
$$

With this formula, one easily verifies that the inverse of $\phi=\left(\mathbb{1}_{L}, \lambda_{2}, \lambda_{3}, \ldots\right)$ is of the form $\phi^{-1}=\left(\mathbb{1}_{L},-\lambda_{2},+2 \lambda_{3}, \ldots\right)$.

The twisting of the trivial $C_{\infty}$-algebra $\mathcal{C}_{\Delta}$ by $\phi=\left(1_{L}, \lambda_{2}, \lambda_{3}, \ldots\right)$ leads to the following formulas for $c_{k}^{\Delta}=\Delta_{k}^{\phi}$; we for clarity omit the Koszul signs:

$$
\begin{aligned}
c_{1}^{\Delta}(v)= & \Delta(v), \\
c_{2}^{\Delta}\left\{v_{1}, v_{2}\right\}= & \Delta\left[v_{1}, v_{2}\right]-\left[\Delta v_{1}, v_{2}\right]-\left[v_{1}, \Delta v_{2}\right], \\
c_{3}^{\Delta}\left\{v_{1},\left\{v_{2}, v_{3}\right\}\right\}= & \Delta\left[v_{1},\left[v_{2}, v_{3}\right]\right]-2\left[v_{1}, \Delta\left[v_{2}, v_{3}\right]\right]+\left[\Delta v_{2},\left[v_{3}, v_{1}\right]\right] \\
& +\left[v_{2}, \Delta\left[v_{3}, v_{1}\right]\right]+\left[\Delta v_{3},\left[v_{1}, v_{2}\right]\right]+\left[v_{3}, \Delta\left[v_{1}, v_{2}\right]\right] \\
& +2\left(\left[v_{1},\left[\Delta\left(v_{2}\right), v_{3}\right]\right]+\left[v_{1},\left[v_{2}, \Delta\left(v_{3}\right)\right]\right]\right) .
\end{aligned}
$$

It is easy to verify that

$$
c_{3}^{\Delta}\left\{v_{1},\left\{v_{2}, v_{3}\right\}\right\}=-2\left[v_{1}, c_{2}^{\Delta}\left\{v_{2}, v_{3}\right\}\right]-c_{2}^{\Delta}\left\{v_{2},\left[v_{3}, v_{1}\right]\right\}-c_{2}^{\Delta}\left\{v_{3},\left[v_{1}, v_{2}\right]\right\}
$$

Therefore, if $\Delta$ is a derivation of the Lie algebra $L$, i.e. if $c_{2}^{\Delta}=0, c_{3}^{\Delta}$ vanishes as expected.

We saw in the $A_{\infty}$ - resp. $L_{\infty}$-cases that the twisting by the automorphism whose components were the canonical maps $\mu_{k}: \mathbb{T}^{k} A \rightarrow A$ resp. $\mu_{k}: \mathbb{S}^{k} A \rightarrow A$, lead to the (unique) braces giving a sensible notion of higher-order derivations. This justifies:

Conjecture The twisting of $\mathcal{C}_{\Delta}=(L, \Delta, 0,0, \ldots)$ by $\phi=\left(1_{L}, \lambda_{2}, \lambda_{3}, \ldots\right)$ gives rise to $C_{\infty}$-braces satisfying the analogs of conditions (3)-(5).

Verifying this conjecture of course depends on describing the isomorphism

$$
\mathbb{L} L \cong \mathbb{L}^{c} L .
$$

\subsection{Other cases}

Let us finish this part by formulating the most general context in which our approach may work. We will need the language of operads for which we refer for instance to $[12,13]$. Let $\mathcal{P}$ be a quadratic Koszul operad and $A$ a $\mathcal{P}$-algebra. Denote by $\mathcal{P}$ ! the Koszul (quadratic) dual of $\mathcal{P}$ [13, Def. II.3.37] and by $\mathbb{F}_{\mathcal{P}} A$ (resp. $\mathbb{F}_{\mathcal{P}}^{c} A$ ) the free 
$\mathcal{P}$-algebra (resp. the cofree conilpotent $\mathcal{P}$-coalgebra) generated (resp. cogenerated) by A.

A strongly homotopy $\mathcal{P}^{!}$-algebra, also called a $\mathcal{P}_{\infty}^{!}$-algebra, is determined by a square-zero coderivation $p$ of $\mathbb{F}_{\mathcal{P}}^{c} A$. If $\Delta$ is a differential on $A$, one has as before the trivial $\mathcal{P}_{\infty}$-algebra $\mathcal{P}_{\Delta}=(A, \Delta, 0,0, \ldots)$ given by extending $\Delta$ to $\mathbb{F}_{\mathcal{P}}^{c} A$.

Under the presence of a natural identification $\mathbb{F}_{\mathcal{P}} A \cong \mathbb{F}_{\mathcal{P}}^{c} A$, one may speak about natural automorphisms that twist $P_{\Delta}$ to $\mathcal{P}_{\infty}^{\text {! }}$-braces. One has the automorphism $\phi$ : $\mathbb{F}_{\mathcal{P}}^{c} A \rightarrow \mathbb{F}_{\mathcal{P}}^{c} A$ whose components are given by the structure map $\mathbb{F}_{\mathcal{P}} A \rightarrow A$. It is sensible to conjecture that the related $\mathcal{P}_{\infty}^{!}$-braces lead to a reasonable notion of higher-order derivations of $\mathcal{P}$-algebras.

In this general set-up, $A_{\infty}$-braces related to associative algebras correspond to the $\mathcal{P}=\mathcal{A}$ ss case, $L_{\infty}$-braces related to commutative associative algebras to $\mathcal{P}=\mathcal{C}$ om, and $C_{\infty}$-braces related to Lie algebras to $\mathcal{P}=\mathcal{L}$ ie, where $\mathcal{A s s}, \mathcal{C}$ om and $\mathcal{L}$ ie denote the operad for associative, commutative associative and Lie algebras, respectively. ${ }^{6}$

\section{Part 2. Naturality and acyclicity}

\section{Naturality}

This section is devoted to natural operations $A^{\otimes k} \rightarrow A$ (resp. $\mathbb{S}^{k} A \rightarrow A$ ), where $A$ is a graded associative (resp. graded commutative associative) algebra with a differential $\Delta$ of degree +1 . Since in the commutative associative case the symmetric group action brings extra complications but nothing conceptually new, we analyze in detail only the associative case.

Associative case. We are going introduce the space $\mathcal{N} a t(k)$ of natural operations $A^{\otimes k} \rightarrow A$ and show that $\mathcal{N} a t(k)$, graded by the degrees of maps and equipped with the differential induced by $\Delta$, is acyclic for each $k \geq 2$. The content of this section is a kindergarten version of the analysis of Deligne's conjecture given in [4].

\subsection{Natural operations}

Intuitively, natural operations $A^{\otimes k} \rightarrow A$ are linear maps composed from the data available for an arbitrary graded associative algebra $A$ with a differential. Equivalently, natural operations are linear combinations of compositions of 'elementary' operations, which are the multiplication, the differential, permutations of the inputs and projections to the homogeneous parts. Our categorial definition given below is chosen so that it excludes the projections; the reason is explained in Exercise 4.8. Our theory can, however, easily be extended to include the projections as well, cf. Exercise 3.7. Let us start with:

Example 3.1 The space $\mathcal{N}$ at (1) of natural operations $A \rightarrow A$ is two-dimensional, spanned by the identity $11: A \rightarrow A \in \mathcal{N}$ at $(1)^{0}$ in degree 0 and $\Delta: A \rightarrow A \in$

$\overline{{ }^{6} \text { Recall that } \mathcal{A} s s^{!} \cong \mathcal{A} \text { sss, } \mathcal{C} o m !} \cong \mathcal{L} i e$ and $\mathcal{L} i e^{!} \cong \mathcal{C}$ om, see e.g. [13, Example II.3.38]. 
$\mathcal{N}$ at $(1)^{1}$ in degree 1 . The space $\mathcal{N}$ at $(2)^{0}$ is spanned by two operations,

$$
a \otimes b \mapsto a b \text { and } a \otimes b \mapsto(-1)^{|a||b|} b a,
$$

where $a b$ resp. $b a$ denotes the product in $A$. The space $\mathcal{N} a t(2)^{1}$ is 6-dimensional, spanned by the operations

$$
a \otimes b \mapsto \Delta(a) b, a \otimes b \mapsto(-1)^{|a|} a \Delta(b), a \otimes b \mapsto \Delta(a b),
$$

and compositions of these operations with the permutation $a \otimes b \mapsto(-1)^{|a||b|} b \otimes a$, i.e. by

$a \otimes b \mapsto(-1)^{|a||b|} \Delta(b) a, a \otimes b \mapsto(-1)^{|b|(|a|+1)} b \Delta(a), a \otimes b \mapsto(-1)^{|a||b|} \Delta(b a)$.

Likewise, $\mathcal{N}$ at $(2)^{2}$ is spanned by

$$
a \otimes b \mapsto \Delta(a) \Delta(b), a \otimes b \mapsto(-1)^{|a|} \Delta(a \Delta(b)), a \otimes b \mapsto \Delta(\Delta(a) b)
$$

and their permutations. Finally, $\mathcal{N}$ at $(2)^{3}$ is two-dimensional, spanned by

$$
a \otimes b \mapsto \Delta(\Delta(a) \Delta(b)) \text { and } a \otimes b \mapsto(-1)^{|b|(|a|+1)} \Delta(\Delta(b) \Delta(a)) .
$$

There are no natural operations $A^{\otimes 2} \rightarrow A$ of degrees $>4$. Observe that the Euler characteristic of the graded space $\mathcal{N}$ at $(2)^{*}$ is $2-6+6-2=0$. This indicates its acyclicity.

All operations $\beta: A^{\otimes 2} \rightarrow A \in \mathcal{N}$ at (2) listed in Example 3.1 share the following property. Let $\left(A, \Delta_{A}\right)$ and $\left(B, \Delta_{B}\right)$ be graded associative algebras with differentials and $\varphi: A \rightarrow B$ a linear map such that

$$
\varphi\left(a^{\prime} a^{\prime \prime}\right)=\varphi\left(a^{\prime}\right) \varphi\left(a^{\prime \prime}\right) \text { and } \varphi \Delta_{A}(a)=\Delta_{B} \varphi(a) \text {, for each } a, a^{\prime}, a^{\prime \prime} \in A \text {. }
$$

Then

$$
\beta\left(\varphi\left(a^{\prime}\right) \otimes \varphi\left(a^{\prime \prime}\right)\right)=\varphi\left(\beta\left(a^{\prime} \otimes a^{\prime \prime}\right)\right) \text { for each } a^{\prime}, a^{\prime \prime} \in A .
$$

Let us emphasize that we do not assume the map $\varphi$ to be homogeneous of degree 0 , it can be an arbitrary linear map $A \rightarrow B$ satisfying (18).

Example This example explains why we did not require the homogeneity of the map $\varphi$ in (18). Consider the operation $\beta: A^{\otimes 2} \rightarrow A$ defined by

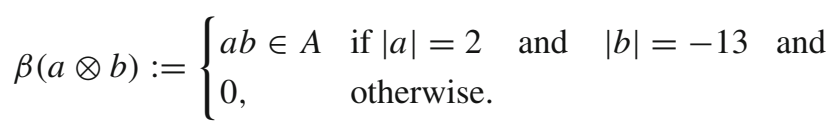

It is certainly 'natural' in that it is defined using the data that are available for any graded associative algebra, but we do not want to consider this type of operation. ${ }^{7}$

\footnotetext{
7 Although, as indicated in Exercise 3.7, we can extend our theory to include also operations of this type.
} 
What excludes $\beta$ from the family of well-behaved operations is precisely the lack of naturality (19) with respect to non-homogeneous maps.

To see why it is so, take $A:=\mathbb{T}(a, b)$, the tensor algebra on two generators with $|a|:=2$ and $|b|:=-13$, and $B:=\mathbb{T}(u, v)$ generated by $u, v$ with $|u|=|v|:=0$. There clearly exists a unique 'non-homogeneous' homomorphism $\varphi: A \rightarrow B$ such that $\varphi(a)=u$ and $\varphi(b)=v .^{8}$ But then

$$
0=\beta(u \otimes v)=\beta(\varphi(a) \otimes \varphi(b)) \neq \varphi(\beta(a \otimes b))=\varphi(a b)=\varphi(a) \varphi(b)=u v,
$$

so $\beta$ is not natural with respect to our extended notion of a homomorphism, though it is still natural with respect to conventional homomorphisms as can be easily checked.

Natural operations thus appear as natural transformations $\beta: \bigotimes^{k} \rightarrow \square$ from the tensor power functor $\otimes^{k}: \operatorname{Algs}^{\Delta} \rightarrow$ Vect to the forgetful functor $\square: \operatorname{Algs}^{\Delta} \rightarrow$ Vect, where $\operatorname{Algs}^{\Delta}$ is the category of graded associative algebras with a differential, with morphisms as in (18), and Vect the category of vector spaces. We however prefer a more explicit:

Definition 3.2 For $k \geq 1$, let $\mathcal{N} a t(k)$ be the abelian group of families of linear maps

$$
\beta_{A}: A^{\otimes k} \rightarrow A
$$

indexed by graded associative algebras $A=(A, \Delta)$ with a differential such that, for any linear map $\varphi: A \rightarrow B$ satisfying (18), the diagram

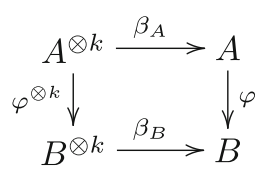

commutes.

We are going to prove a structure theorem for natural operations. Denote by $\mathcal{F} r\left(x_{1}, \ldots, x_{k}\right)$ the free graded associative algebra with a differential, generated by degree 0 elements $x_{1}, \ldots, x_{k}$ (an explicit description is given in §3.2). Denote also by $\mathcal{F} r_{1, \ldots, 1}\left(x_{1}, \ldots, x_{k}\right)$ the subspace of $\mathcal{F} r\left(x_{1}, \ldots, x_{k}\right)$ spanned by the words that contain each generator precisely once.

Proposition 3.3 For each $k \geq 1$ one has a natural isomorphism

$$
\xi: \mathcal{N} a t(k) \cong \mathcal{F} r_{1, \ldots, 1}\left(x_{1}, \ldots, x_{k}\right) .
$$

Proof Denote, for brevity, $\mathcal{F} r:=\mathcal{F} r\left(x_{1}, \ldots, x_{k}\right)$. Let $A=(A, \Delta)$ be an arbitrary algebra with a differential. Given elements $a_{1}, \ldots, a_{k} \in A$, there exists a unique $\Phi_{a_{1}, \ldots, a_{n}}^{A}: \mathcal{F} r \rightarrow A$ satisfying (18), specified by requiring $\Phi_{a_{1}, \ldots, a_{n}}^{A}\left(x_{i}\right):=a_{i}$ for $1 \leq i \leq k$.

\footnotetext{
8 The reason is that $\mathbb{T}(a, b)$ is free also in the category of ungraded associative algebras.
} 
Given a natural operation $\beta \in \mathcal{N} a t(k)$ and $a_{1}, \ldots, a_{k} \in A$, one has the commutative diagram

$$
\begin{aligned}
\mathcal{F} r & \otimes k \\
\left(\Phi_{a_{1}, \ldots, a_{k}}^{A}\right)^{\otimes k} \downarrow & \stackrel{\beta_{\mathcal{F} r}}{\longrightarrow} \mathcal{F r} \\
A^{\otimes k} \stackrel{\beta_{A}}{\longrightarrow} & \downarrow^{\longrightarrow} \Phi_{a_{1}, \ldots, a_{k}}^{A}
\end{aligned}
$$

by naturality (20). We will also need the particular case of (21) when $A=\mathcal{F} r$ and $a_{i}=u_{i} x_{i}$, for some scalars $u_{i} \in \mathbb{k}, 1 \leq i \leq k$ :

$$
\begin{aligned}
\mathcal{F} r & \stackrel{\beta_{\mathcal{F} r}}{\longrightarrow} \mathcal{F} r \\
\left(\Phi_{u_{1} x_{1}, \ldots, u_{k} x_{k}}^{A}\right)^{\otimes k} \downarrow & \\
\mathcal{F}^{\otimes k} \stackrel{\beta_{\mathcal{F} r}}{\longrightarrow} & \mathfrak{F}^{\longrightarrow} \Phi_{u_{1} x_{1}, \ldots, u_{k} x_{k}}^{A} .
\end{aligned}
$$

Recall that $\mathcal{F} r_{1, \ldots, 1}:=\mathcal{F} r_{1, \ldots, 1}\left(x_{1}, \ldots, x_{k}\right)$ denotes the subspace of elements containing each generator $x_{1}, \ldots, x_{k}$ precisely once. We begin the actual proof by observing that each natural operation $\beta \in \mathcal{N}$ at $(k)$ determines an element $\xi(\beta) \in \mathcal{F} r$ by

$$
\xi(\beta):=\beta_{\mathcal{F} r}\left(x_{1} \otimes \cdots \otimes x_{n}\right) \in \mathcal{F} r
$$

We will show that, quite miraculously, $\xi(\beta)$ belongs to $\mathcal{F} r_{1, \ldots, 1}$. Clearly, $\mathcal{F} r$ decomposes as

$$
\mathcal{F} r=\bigoplus_{j_{1}, \ldots, j_{k} \geq 0} \mathcal{F} r_{j_{1}, \ldots, j_{k}}
$$

where $\mathcal{F} r_{j_{1}, \ldots, j_{k}} \subset \mathcal{F} r$ is the subspace of elements having precisely $j_{i}$ instances of $x_{i}$ for each $1 \leq i \leq k$. The endomorphism $\Phi_{u_{1} x_{1}, \ldots, u_{k} x_{k}}^{A}: \mathcal{F} r \rightarrow \mathcal{F} r$ acts on $\mathcal{F} r_{j_{1}, \ldots, j_{k}}$ by the multiplication with $u_{1}^{j_{1}} \cdots u_{k}^{j_{k}}$; the subspace $\mathcal{F} r_{j_{1}, \ldots, j_{k}} \subset \mathcal{F} r$ is, in fact, characterized by this property. The element $\xi(\beta)$ uniquely decomposes as $\xi(\beta)=\sum_{j_{1}, \ldots, j_{k} \geq 0} \xi(\beta)_{j_{1}, \ldots, j_{k}}$, for some $\xi(\beta)_{j_{1}, \ldots, j_{k}} \in \mathcal{F} r_{j_{1}, \ldots, j_{k}}$.

Let us turn our attention to (22). By the definition of the map $\Phi_{u_{1} x_{1}, \ldots, u_{k} x_{k}}^{A}$, one has

$$
\beta_{\mathcal{F} r}\left(\left(\Phi_{u_{1} x_{1}, \ldots, u_{k} x_{k}}^{A}\right)^{\otimes k}\right)\left(x_{1} \otimes \cdots \otimes x_{k}\right)=\beta_{\mathcal{F} r}\left(u_{1} x_{1} \otimes \cdots \otimes u_{k} x_{k}\right)
$$

while the linearity of $\beta_{\mathcal{F} r}$ implies

$$
\beta_{\mathcal{F} r}\left(u_{1} x_{1} \otimes \cdots \otimes u_{k} x_{k}\right)=u_{1} \cdots u_{k} \cdot \beta_{\mathcal{F} r}\left(x_{1} \otimes \cdots \otimes x_{k}\right)=u_{1} \cdots u_{k} \cdot \xi(\beta) .
$$

On the other hand

$$
\begin{aligned}
& \Phi_{u_{1} x_{1}, \ldots, u_{k} x_{k}}^{A}\left(\beta_{\mathcal{F} r}\right)\left(x_{1} \otimes \cdots \otimes x_{k}\right) \\
& \quad=\Phi_{u_{1} x_{1}, \ldots, u_{k} x_{k}}^{A}(\xi(\beta))=\sum_{j_{1}, \ldots, j_{k} \geq 0} u_{1}^{j_{1}} \cdots u_{k}^{j_{k}} \cdot \xi(\beta)_{j_{1}, \ldots, j_{k}}
\end{aligned}
$$


therefore the commutativity of (22) means that

$$
u_{1} \cdots u_{k} \cdot \xi(\beta)=\sum_{j_{1}, \ldots, j_{n} \geq 0} u_{1}^{j_{1}} \cdots u_{k}^{j_{k}} \cdot \xi(\beta)_{j_{1}, \ldots, j_{k}}
$$

for each $u_{1}, \ldots, u_{k} \in \mathbb{k}$. We conclude that $\xi(\beta)_{j_{1}, \ldots, j_{k}}=0$ if $\left(j_{1}, \ldots, j_{k}\right) \neq(1, \ldots, 1)$, so $\xi(\beta)=\xi(\beta)_{1, \ldots, 1} \in \mathcal{F} r_{1, \ldots, 1}$ as claimed.

We leave as an exercise to prove that, vice versa, each element $\xi \in \mathcal{F} r_{1, \ldots, 1}$ determines a natural operation $\beta(\xi) \in \mathcal{N}$ at $(k)$ by the formula

$$
\beta(\xi)_{A}\left(a_{1} \otimes \cdots \otimes a_{k}\right)=\Phi_{a_{1}, \ldots, a_{k}}^{A}(\xi), \text { for each } a_{1}, \ldots, a_{k} \in A
$$

$\mathrm{cf}$. the proof of [4, Proposition 2.9]. The above constructions define mutually inverse correspondences $\beta \mapsto \xi(\beta)$ and $\xi \mapsto \beta(\xi)$ that give the isomorphism of the proposition.

Remark A crucial step of the previous proof was that $\xi(\beta)$ belonged to $\mathcal{F} r_{1, \ldots, 1}$. It was implied by the multilinearity, which is a particular feature of the monoidal structure given by $\otimes$. In the cartesian situation, $\xi(\beta)$ might have been an arbitrary element of the free algebra $\mathcal{F} r\left(x_{1}, \ldots, x_{k}\right)$.

\subsection{The algebra $\mathcal{F} r\left(x_{1}, \ldots, x_{k}\right)$.}

Elements of the free algebra $\mathcal{F} r=\mathcal{F} r\left(x_{1}, \ldots, x_{k}\right)$ are results of iterated applications of the associative multiplication and the differential on the generators $x_{1}, \ldots, x_{k}$. The subspace $\mathcal{F} r_{1, \ldots, 1}=\mathcal{F} r_{1, \ldots, 1}\left(x_{1}, \ldots, x_{k}\right)$ is spanned by words containing each generator precisely once. A typical element of $\mathcal{F} r_{1, \ldots, 1}$ is thus an expression as

$$
\Delta\left(\Delta\left(x_{3}\right) x_{6} \Delta\left(x_{4}\right)\right) x_{5} \Delta\left(x_{2} x_{1}\right)
$$

The algebra $\mathcal{F} r$ and thus also $\mathcal{F} r_{1, \ldots, 1}$ is graded by the number of occurrences of $\Delta$; the element in (25) therefore belongs to $\mathcal{F} r_{1, \ldots, 1}^{4}$.

We can clearly encode elements of $\mathcal{F} r_{1, \ldots, 1}$ by 'flow diagrams' that record how the multiplication and the differential are applied. For instance, the diagram encoding (25) is

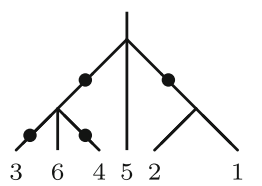

Its underlying graph is a rooted (=oriented) planar tree with the root pointing upwards. The labels of its leaves (=inputs) mark the position of the generators. The vertices symbolize iterated multiplication while the bullets the application of the differential.

We see that elements of $\mathcal{F} r_{1, \ldots, 1}\left(x_{1}, \ldots, x_{k}\right)$ can be represented by linear combinations of planar rooted trees $T$ such that 
- each vertex of $T$ has at least two inputs,

- all internal edges and possibly some external edges are decorated by the bullet $\bullet$, and

- the leaves of $T$ are labelled by a permutation of $(1, \ldots, k)$.

Let us denote by $\mathcal{T}(k)$ the set of all trees as above. Proposition 3.3 together with our description of $\mathcal{F} r_{1, \ldots, 1}\left(x_{1}, \ldots, x_{k}\right)$ gives

Corollary 3.4 For each $k \geq 1$ one has a natural isomorphism $\mathcal{N}$ at $(k) \cong \operatorname{Span}$ $(\mathcal{T}(k))$.

It follows from general theory [13, Proposition II.1.27] that $\mathcal{N a t}(k)$ is the arity $k$-th piece of the operad $\mathcal{N} a t$ whose algebras are couples $(A, \Delta)$ consisting of an associative algebra and a differential. We will, however, not need this interpretation in the sequel.

Example Corollary 3.4 offers the following description of $\mathcal{N}$ at (2):

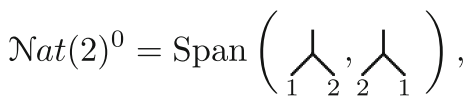

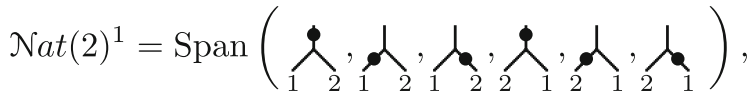

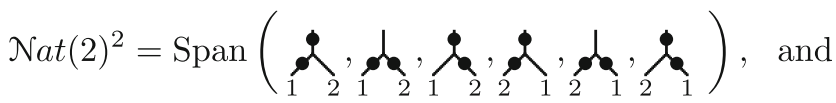

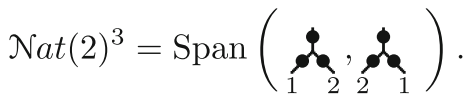

We leave as an exercise to relate the above description to the operations listed in Example 3.1.

Example 3.5 It easily follows from Corollary 3.4 that $\mathcal{N}$ at $(k)^{0}$ is spanned by operations

$$
\beta\left(a_{1}, \ldots, a_{k}\right)=\varepsilon(\sigma) \cdot a_{\sigma(1)} \cdots a_{\sigma(k)}, \sigma \in \Sigma_{n},
$$

i.e. $\mathcal{N}$ at $(k)^{0} \cong \mathbb{k}\left[\Sigma_{n}\right]$ for $k \geq 1$.

\subsection{Acyclicity}

There is a differential on $\mathcal{N} a t(k)$ induced by $\Delta$. For $\beta: A^{\otimes k} \rightarrow A$ it is defined by

$$
\delta(\beta):=\Delta \beta-(-1)^{|\beta|} \sum_{1 \leq i \leq k} \beta\left(\mathbb{1}^{\otimes(i-1)} \otimes \Delta \otimes \mathbb{1}^{\otimes(k-i)}\right) .
$$

When evaluating the above formula, we shall of course take into account the Koszul sign convention. For instance, if $\beta \in \mathcal{N}$ at (2) and $a, b, \in A$, then

$$
\delta(\beta)(a \otimes b)=\Delta \beta(a \otimes b)-(-1)^{|\beta|} \beta(\Delta(a) \otimes b)-(-1)^{|a|+|\beta|} \beta(a \otimes \Delta(b)) .
$$


It is simple to verify that $\delta^{2}=0$, so $\left(\mathcal{N} a t(k)^{*}, \delta\right)$ is a cochain complex. We leave as an exercise to describe $\delta$ in terms of trees.

Proposition 3.6 The cochain complex

$$
\mathrm{k}=\mathcal{N} a t(k)^{0} \stackrel{\delta}{\longrightarrow} \mathcal{N} a t(k)^{1} \stackrel{\delta}{\longrightarrow} \mathcal{N} a t(k)^{2} \stackrel{\delta}{\longrightarrow} \cdots
$$

is acyclic for each $k \geq 2$. In particular, the map $\delta: \mathbb{k}_{k}=\mathcal{N}$ at $(k)^{0} \rightarrow \mathcal{N}$ at $(k)^{1}$ is monic.

The case of $k=1$ is a particular one, as the differential $\delta: \mathcal{N}$ at $(1)^{0} \rightarrow \mathcal{N}$ at $(1)^{1}$ is the zero map $\operatorname{Span}(11) \stackrel{0}{\rightarrow} \operatorname{Span}(\Delta)$. The explanation is that the identity $11: A \rightarrow A$ is the only natural operation that is 'generically' a chain map.

Proof of Proposition 3.6 We describe a contracting homotopy. By Corollary 3.4, each natural operation $\beta \in \mathcal{N} a t(k)$ is represented by a unique linear combination of trees from $\mathcal{T}(k)$. It is therefore enough to specify how the homotopy acts on operations given by a single tree.

Let $\beta$ be represented by $T \in \mathcal{T}(k)$. If the root edge of $T$ is decorated by the bullet, we define $h(\beta)$ as the operation represented the tree $T^{\prime}$ obtained from $T$ by removing the decoration of the root. We define $h(\beta):=0$ if the root edge of $T$ is not decorated by the bullet. We leave as an exercise to verify that $h \delta+\delta h=\mathbb{1}$, so that $h$ is a contracting homotopy.

Notice that the complex $\mathcal{N} a t(k)$ is isomorphic to the direct sum of $k$ ! copies of the contractible subcomplex $\underline{\mathcal{N} a t}(k) \subset \mathcal{N} a t(k)$ consisting of operations represented by trees with leaves indexed by the identity permutation $(1, \ldots, k)$.

Exercise 3.7 Let $c \mathcal{N} a t(k)$ be the abelian group of families of linear maps $\beta_{A}$ : $A^{\otimes k} \rightarrow A$ such that the diagram (20) commutes for all homogeneous $\varphi$ 's. ${ }^{9}$ Prove that then

$$
c \mathcal{N} a t(k) \cong \bigoplus_{d_{1}, \ldots, d_{k}} c \mathcal{N} a t\left(d_{1}, \ldots, d_{k}\right)
$$

where $c \mathcal{N} a t\left(d_{1}, \ldots, d_{k}\right)$ consists of $\beta_{A}$ 's such that

$$
\beta_{A}\left(a_{1}, \ldots, a_{k}\right) \neq 0 \text { only if }\left(\left|a_{1}\right|, \ldots,\left|a_{k}\right|\right)=\left(d_{1}, \ldots, d_{k}\right) .
$$

Prove, moreover, that $c \mathcal{N} a t\left(d_{1}, \ldots, d_{k}\right) \cong \mathcal{N} a t(k)$ for each $d_{1}, \ldots, d_{k}$, and discuss the acyclicity of $c \mathcal{N} a t(k)$.

\footnotetext{
9 The notation $c \mathcal{N}$ at $(k)$ refers to colored natural operations.
} 


\subsection{Natural $A_{\infty}$-algebras}

Let $(A, \Delta)$ be a graded associative algebra with a differential. We call an $A_{\infty}$-algebra $\mathcal{A}=\left(A, m_{1}, m_{2}, \ldots\right)$ natural if $m_{k}: A^{\otimes k} \rightarrow A$ are natural operations from $\mathcal{N}$ at $(k)^{1}$ for each $k \geq 1$. Formally, a natural $A_{\infty}$-algebra should be considered as a family $\left\{\mathcal{A}_{(A, \Delta)}\right\}$ of $A_{\infty}$-algebras indexed by algebras with a differential, such that each $\varphi$ as in (18) induces a strict morphism $\mathcal{A}_{\left(A, \Delta_{A}\right)} \rightarrow \mathcal{A}_{\left(B, \Delta_{B}\right)}$. We however believe that our simplification will not lead to confusion.

An automorphism $\phi: \mathbb{T}^{c} A \rightarrow \mathbb{T}^{c} A$ is natural if all its components $\phi_{k}: A^{\otimes k} \rightarrow A$ are natural operations from $\mathcal{N}$ at $(k)^{0}$. Natural automorphisms with $\phi_{1}=\mathbb{1}_{A}$ form a monoid $\operatorname{Aut}(A)$. It is clear that the twisting of a natural $A_{\infty}$-algebra by a natural automorphism is a natural $A_{\infty}$-algebra.

Example 3.8 It follows from Example 3.5 that natural automorphisms $\phi \in \operatorname{Aut}(A)$ are encoded by sequences $\left(\mathbb{1}_{A}, \omega_{2}, \omega_{3}, \ldots\right)$ of elements $\omega_{k} \in \mathbb{k}\left[\Sigma_{k}\right]$. One has an important submonoid $\operatorname{Aut}(A) \subset \operatorname{Aut}(A)$ of sequences such that all $\omega_{k}$ 's are the identities $\mathbb{1}_{\Sigma_{k}}$.

Exercise 3.9 Assume that $\Delta$ is a derivation. The twisting of $\mathcal{A}_{\Delta}=(A, \Delta, 0,0, \ldots)$ by an arbitrary natural automorphism is then strictly isomorphic to $\mathcal{A}_{\Delta}$, i.e. $\Delta^{\phi}=\Delta$.

Commutative associative case. We are going to formulate commutative versions of the main statements from the first part of this section. We omit the proofs which are analogous to the non-commutative case.

Let $A$ be a graded commutative associative algebra with a differential $\Delta$. Natural operations are, analogously to Definition 3.2, natural transformations $\beta_{A}: \mathbb{S}^{k} A \rightarrow A$ from the $k$ th symmetric power of $A$ to $A$. Let us denote by $\mathcal{N} a t(k)$ the abelian group of all these natural operations. To see how $\mathcal{N} a t(k)$ differs from its non-commutative counterpart, we give commutative versions of Examples 3.1 and 3.5.

Example 3.10 (Commutative version of Example 3.1) The space $\mathcal{N}$ at (1) is, as in the non-commutative case, spanned by the identity $11: A \rightarrow A$ in degree 0 and $\Delta: A \rightarrow A$ in degree 1 . The space $\mathcal{N} a t(2)^{0}$ is spanned by the multiplication $a \odot b \mapsto a b$, with $\odot$ denoting the symmetric product. The space $\mathcal{N}$ at $(2)^{1}$ is two-dimensional, spanned by the operations

$$
a \odot b \mapsto \Delta(a) b+(-1)^{|a||b|} \Delta(b) a \text { and } a \odot b \mapsto \Delta(a b) .
$$

Likewise, $\mathcal{N}$ at $(2)^{2}$ is spanned by

$$
a \odot b \mapsto \Delta(a) \Delta(b) \text { and } a \odot b \mapsto \Delta(\Delta(a) b)-(-1)^{|a||b|} \Delta(\Delta(b) a)
$$

Finally, $\mathcal{N}$ at $(2)^{3}$ is spanned by

$$
a \odot b \mapsto \Delta(\Delta(a) \Delta(b)) .
$$

The Euler characteristic of the graded space $\mathcal{N}$ at $(2)^{*}$ is $1-2+2-1=0$, so the acyclicity can be expected as in the non-commutative case. 
Example 3.11 (Commutative version of Example 3.5) The space $\mathcal{N a t}(k)^{0}$ is onedimensional, spanned by the iterated multiplication $\mu_{k}\left(a_{1}, \ldots, a_{k}\right)=a_{1} \cdots a_{k}$, so $\mathcal{N}$ at $(k)^{0} \cong \mathbb{k}$ for each $k \geq 1$.

An obvious modification of Proposition 3.3 holds, with $\mathcal{F} r\left(x_{1}, \ldots, x_{k}\right)$ this time the free commutative associative algebra. Corollary 3.4 holds as well, with $\mathcal{T}(k)$ replaced by the space of all 'abstract,' i.e. non-planar, trees. As in the non-commutative case, $\Delta$ induces a differential $\delta$ so that $\left(\mathcal{N} a t(k)^{*}, \delta\right)$ is acyclic for each $k \geq 2$.

The notions of a natural $L_{\infty}$-algebras and natural automorphisms $\phi: \mathbb{S}^{c}(A) \rightarrow$ $\mathbb{S}^{c}(A)$ translate verbatim. The following example however shows that the space $\operatorname{Aut}(A)$ of natural automorphisms is much smaller than in the non-commutative case.

Example 3.12 The description of $\mathcal{N}$ at $(k)^{0}$ given in Example 3.11 implies that natural automorphisms $\phi \in \operatorname{Aut}(A)$ are encoded by sequences $\left(\mathbb{1}_{A}, f_{2}, f_{3}, \ldots\right)$ of scalars $f_{k} \in \mathbb{k}$.

\section{Main results}

We are going to formulate and prove the main theorems. As in Sect. 3, we treat in detail only the associative non-commutative case.

Associative case. Let $A$ be a graded associative algebra with a differential $\Delta$, and $\mathcal{A}_{\Delta}=(A, \Delta, 0,0, \ldots)$ the trivial $A_{\infty}$-algebra of Example 2.2. According to the following result, each natural $A_{\infty}$-algebra whose linear operation $m_{1}$ equals $\Delta$, is uniquely given by a twisting of $\mathcal{A}_{\Delta}$, see $\$ 3.4$ and Definition 2.3 for the meaning of naturality and twisting. In particular, each such an $A_{\infty}$-algebra is (weakly) isomorphic to $\mathcal{A}_{\Delta}$.

Theorem 4.1 For each natural $A_{\infty}$-algebra $\mathcal{A}=\left(A, m_{1}, m_{2}, m_{3}, \ldots\right)$ such that

$$
m_{1}=\Delta
$$

there exist a unique natural automorphism $\phi=\left(\mathbb{1}_{A}, \phi_{2}, \phi_{3}, \ldots\right)$ of the tensor coalgebra $\mathbb{T}^{c} A$ such that $\mathcal{A}$ equals the twisting of $\mathcal{A}_{\Delta}$ via $\phi$. Explicitly

$$
m_{k}\left(a_{1}, \ldots, a_{k}\right)=\pi \phi^{-1} \Delta \phi\left(a_{1}, \ldots, a_{k}\right), \text { for } a_{n}, \ldots, a_{k} \in A
$$

where $\pi: \mathbb{T}^{c} A \rightarrow A$ is the canonical projection.

The $A_{\infty}$-algebra $\mathcal{A}=\left(A, m_{1}, m_{2}, m_{3}, \ldots\right)$ satisfies also

$$
m_{2}\left(a_{1}, a_{2}\right)=\Delta\left(a_{1} a_{2}\right)-(-1)^{\left|a_{1}\right|} a_{1} \Delta\left(a_{2}\right)-\Delta\left(a_{1}\right) a_{2}, a_{1}, a_{2} \in A,
$$

if and only if the $\phi_{2}$-part of the automorphism $\phi$ equals the product of $A$, that is if $\phi_{2}(a, b)=$ ab for each $a, b \in A$.

Proof Let $m$ denote the coderivation of $\mathbb{T}^{c} A$ determined by $\left(m_{1}, m_{2}, m_{3}, \ldots\right)$. Assume that we have already constructed an automorphism $\vartheta=\left(\mathbb{1}_{A}, \vartheta_{2}, \vartheta_{3}, \ldots\right)$ of $\mathbb{T}^{c} A$ such that 


$$
\left(\vartheta m \vartheta^{-1}\right)_{1}=\Delta \text { and }\left(\vartheta m \vartheta^{-1}\right)_{k}=0, \text { for } 1<k \leq n
$$

with some $n \geq 1$. To simplify the notation, denote $\mu:=\vartheta m \vartheta^{-1}$. The coderivation $\mu$ determines an $A_{\infty}$-structure of the form $\left(A, \Delta, 0, \ldots, 0, \mu_{n+1}, \ldots\right)$. Axiom (7) for $n+1$ implies that $\delta\left(\mu_{n+1}\right)=0$. Consider any automorphism $\alpha$ of $\mathbb{T}^{c} A$ of the form $\alpha=\left(\mathbb{1}_{A}, 0, \ldots, 0, \alpha_{n+1}, \ldots\right)$. Clearly, $\left(\alpha \mu \alpha^{-1}\right)_{1}=\Delta,\left(\alpha \mu \alpha^{-1}\right)_{k}=0$ for $1<k \leq n$ and

$$
\begin{aligned}
\left(\alpha \mu \alpha^{-1}\right)_{n+1} & =\mu_{n+1}+\sum_{1 \leq j \leq n+1} \alpha_{n+1}\left(\mathbb{1}_{A}^{\otimes(j-1)} \otimes \Delta \otimes \mathbb{1}_{A}^{\otimes(n-j+1)}\right)-\Delta \alpha_{n+1} \\
& =\mu_{n+1}-\delta\left(\alpha_{n+1}\right) .
\end{aligned}
$$

Since $\delta\left(\mu_{n+1}\right)=0$, by Proposition 3.6 one finds $\alpha_{n+1}$ such that $\mu_{n+1}=\delta\left(\alpha_{n+1}\right)$. With this choice, $\left(\alpha \mu \alpha^{-1}\right)_{n+1}=0$, thus $\phi^{\prime}:=\alpha \phi$ satisfies

$$
\left(\phi^{\prime} m \phi^{-1}\right)_{1}=\Delta \text { and }\left(\phi^{\prime} m \phi^{\prime-1}\right)_{k}=0 \text {, for } 1<k \leq n+1 \text {. }
$$

This shows that we can inductively construct an automorphism $\phi$ of $\mathbb{T}^{c} A$ such that $\phi m \phi^{-1}=\Delta$ or, equivalently, $m=\phi^{-1} \Delta \phi$. The first part of the theorem is proven.

To demonstrate that the twisting automorphism is unique, assume that $m=$ $\phi^{-1} \Delta \phi=\psi^{-1} \Delta \psi$. Then $\omega:=\phi \psi^{-1}$ satisfies $\omega^{-1} \Delta \omega=\Delta$. For the bilinear part $\omega_{2}$ of $\omega$ this gives

$$
\omega_{2}\left(\Delta \otimes \mathbb{1}_{A}\right)+\omega_{2}\left(\mathbb{1}_{A} \otimes \Delta\right)-\Delta \omega_{2}=0
$$

i.e. $\delta\left(\omega_{2}\right)=0$. Since $\omega \in \mathcal{N}$ at $(k)^{0}, \omega_{2}=0$ by Proposition 3.6. In the same vein we prove inductively that $\omega_{k}=0$ for all $k \geq 2$, therefore $\omega=\mathbb{1}: \mathbb{T}^{c} A \rightarrow \mathbb{T}^{c} A$ so $\phi=\psi$.

Theorem 4.1 combined with Example 3.8 gives:

Corollary 4.2 Natural $A_{\infty}$-algebras satisfying (27) are parametrized by power series

$$
\phi(t):=t+\omega_{2} t^{2}+\omega_{3} t^{3}+\omega_{4} t^{4}+\cdots
$$

with $\omega_{k} \in \mathbb{k}\left[\Sigma_{k}\right], k \geq 2$. Natural $A_{\infty}$-algebras satisfying also (28) are parametrized by expressions (29) with $\omega_{2}$ the identity permutation $\mathbb{1}_{\Sigma_{2}}$.

A weak isomorphism of $A_{\infty}$-algebras induces a strict isomorphism of their cohomology algebras. We therefore get another

Corollary The cohomology $H^{*}(A, \Delta)$ of any natural $A_{\infty}$-algebra $\left(A, \Delta, m_{2}, m_{3}, \ldots\right)$ is the trivial associative algebra with the underlying space $H^{*}(A, \Delta)$.

The observation made in Example 3.9 combined with Theorem 4.1 lead to another Corollary Assume that $\Delta$ is a derivation of $A$. Then any natural $A_{\infty}$-braces vanish on $A$. 
We finally formulate our characterization of Börjeson braces [6] recalled in Example 1.3.

Theorem 4.3 Börjeson's braces $\left(A, \Delta, b_{2}^{\Delta}, b_{3}^{\Delta}, b_{4}^{\Delta}, \ldots\right)$ are the unique, up to a strict isomorphism, natural recursive $A_{\infty}$-braces defined over $\mathbb{Z}$ such that

(i) $b_{2}^{\Delta}$ measures the deviation of $\Delta$ from being a derivation, i.e.

$$
b_{2}^{\Delta}\left(a_{1}, a_{2}\right)=\Delta\left(a_{1} a_{2}\right)-\Delta\left(a_{1}\right) a_{2}-(-1)^{\left|a_{1}\right|} a_{1} \Delta\left(a_{2}\right), \quad a_{1}, a_{2} \in A,
$$

(ii) the coefficient at $\Delta\left(a_{1} a_{2} a_{3}\right)$ in $b_{3}^{\Delta}\left(a_{1}, a_{2}, a_{3}\right)$ is either +1 or -1 and,

(iii) the hereditarity is satisfied, that is for all $k \geq 1$,

$$
b_{k}^{\Delta}=0 \text { implies } b_{k+1}^{\Delta}=0 .
$$

It is obvious that the Börjeson braces are recursive and satisfy (i) and (ii). Their hereditarity established in [6] follows from an inductive formula mentioned in Remark 4.6. It remains to prove that (i)-(iii) characterize Börjeson's braces up to a strict isomorphism. This will follow from Propositions 4.4 and 4.5 below.

Proposition 4.4 Suppose that $A=\left(A, \Delta, m_{2}, m_{3}, m_{4}, \ldots\right)$ are natural hereditary $A_{\infty}$-braces such that $m_{2}=b_{2}^{\Delta}$ and $m_{3}=b_{3}^{\Delta}$. Then $m_{k}=b_{k}^{\Delta}$ for any $k \geq 2$.

Proof Assume we have already proved that

$$
b_{k}^{\Delta}=m_{k} \text { for } 2 \leq k \leq n,
$$

with some $n \geq 3$. The $A_{\infty}$-axiom (7) taken with $n=k+1$ implies that $\delta\left(m_{n+1}\right)=$ $\delta\left(b_{n+1}^{\Delta}\right)$, i.e. $\delta\left(m_{n+1}-b_{n+1}^{\Delta}\right)=0$. By Proposition 3.6 there exists $z_{n+1} \in \mathcal{N} a t(n+1)^{0}$ such that

$$
m_{n+1}-b_{n+1}^{\Delta}=\delta z_{n+1} .
$$

Consider the free associative algebra $\mathcal{F} r\left(x_{1}, \ldots, x_{n+1}\right)$ on degree 0 variables $x_{1}, \ldots, x_{n+1}$. Let $A_{n+1}$ be $\mathcal{F r}\left(x_{1}, \ldots, x_{n+1}\right)$ quotiented by the ideal $I_{n+1}$ generated by

$$
m_{n}\left(a_{1}, \ldots, a_{n}\right) \text { for } a_{1}, \ldots, a_{n} \in \mathcal{F} r\left(x_{1}, \ldots, x_{n+1}\right) .
$$

Then both $b_{n+1}^{\Delta}$ and $m_{n+1}$ vanish on $A_{n+1}$, since both braces are hereditary. By (30), $\delta z_{n+1}$ must vanish on $A_{n+1}$, too. In particular, $\delta z_{n+1}\left(x_{1}, \ldots, x_{n+1}\right)=0 .{ }^{10}$ We are going to prove that this implies that $z_{n+1}=0$, so $m_{n+1}=b_{n+1}^{\Delta}$ again by (30).

It follows from the description of $\mathcal{N} a t(n+1)^{0}$ given in Example 3.11 that

$$
z_{n+1}\left(a_{1}, \ldots, a_{n+1}\right)=\sum_{\sigma \in \Sigma_{n+1}} \xi_{\sigma} a_{\sigma(1)} a_{\sigma(1)} \cdots a_{\sigma(n+1)}
$$

\footnotetext{
10 As customary, we denote both the generators of $\mathcal{F} r\left(x_{1}, \ldots, x_{n+1}\right)$ and their equivalence classes in $A_{n+1}$ by the same symbols.
} 
with some $\xi_{\sigma} \in \mathbb{k}$, therefore

$$
\begin{aligned}
\delta z_{n+1}\left(x_{1}, \ldots, x_{n+1}\right) & \\
= & \sum_{\sigma \in \Sigma_{n+1}} \xi_{\sigma}\left(\Delta\left(x_{\sigma(1)} \cdots x_{\sigma(n+1)}\right)\right. \\
& \left.-\sum_{1 \leq i \leq n+1} x_{\sigma(1)} \cdots \Delta\left(x_{\sigma(i)}\right) \cdots x_{\sigma(n+1)}\right) .
\end{aligned}
$$

The crucial observation is that modding out by the ideal $I_{n+1}$ generated by (31) does not introduce any relations involving the monomials

$$
\Delta\left(x_{\sigma(1)}\right) x_{\sigma(2)} \cdots x_{\sigma(n+1)}, \sigma \in \Sigma_{n+1} .
$$

Let us show for instance that the degree 1 part $I_{n+1}^{1}$ of the ideal $I_{n+1}$ does not involve the element $\Delta\left(x_{1}\right) x_{2} \cdots x_{n+1}$. It follows from the definition of an ideal that the subspace of $I_{n+1}^{1}$ spanned by words containing $x_{1}, \ldots, x_{n+1}$ in this order consists of linear combinations of the monomials

$x_{1} m_{n}\left(x_{2}, \ldots, x_{n+1}\right), m_{n}\left(x_{1} x_{2}, \ldots, x_{n+1}\right), \ldots, m_{n}\left(x_{1}, \ldots, x_{n} x_{n+1}\right), m_{n}\left(x_{1}, \ldots, x_{n}\right) x_{n+1}$.

Looking at the explicit form of $m_{n}=b_{n}^{\Delta}$ we immediately realize that none of the above terms contains the monomial $\Delta\left(x_{1}\right) x_{2} \cdots x_{n+1}$. The argument for other permutations is similar.

Inspecting the coefficients at the terms (33) in (32), we see that $\delta z_{n+1}=0$ in $A_{n+1}$ only if all $\xi_{\sigma}, \sigma \in \Sigma_{n+1}$, are trivial. So $z_{n+1}=0$ as required, and the induction goes on.

In the proof of Proposition 4.4, the requirement that $n \geq 3$ in (30) was crucial. Indeed, the monomial $\Delta\left(x_{1}\right) x_{2} x_{3}$ does occur in $m_{2}\left(x_{1}, x_{2}\right) x_{3}$, so the argument following formula (33) does not work for $n=2$. We must therefore prove also the following

Proposition 4.5 Let $\mathcal{A}=\left(A, \Delta, m_{2}, m_{3}, m_{4}, \ldots\right)$ be a natural recursive $A_{\infty}$-algebra such that $m_{2}=b_{2}^{\Delta}$, the coefficient $C_{3}$ at $\Delta\left(a_{1} a_{2} a_{3}\right)$ in $m_{3}\left(a_{1}, a_{2}, a_{3}\right)$ is either 1 or -1 , and $m_{3}=0$ implies $m_{4}=0$. Then in fact $C_{3}=1$ and $m_{3}=b_{3}^{\Delta}$.

Proof Boring tour de force. It follows from the assumptions that the automorphism $\phi$ inducing $\mathcal{A}$ must be of the form $\phi=\left(\mathbb{1}_{A}, \mu, C_{3} \mu_{3}, \ldots\right)$. This means that $a_{1}, a_{2}, a_{3}$ appear in $m_{3}$ only in this order. It is simple to prove that $m_{3}$ 's with this property form the family:

$$
\begin{aligned}
m_{3}\left(a_{1}, a_{2}, a_{3}\right)= & (1+\alpha) \Delta\left(a_{1} a_{2} a_{3}\right)-\alpha\left(\left(\Delta\left(a_{1}\right) a_{2} a_{3}+(-1)^{\varepsilon} a_{1} a_{2} \Delta\left(a_{3}\right)\right)\right. \\
& +(1-\alpha)(-1)^{\varepsilon} a_{1} \Delta\left(a_{2}\right) a_{3}-\left(\Delta\left(a_{1} a_{2}\right) a_{3}+(-1)^{\varepsilon} a_{1} \Delta\left(a_{2} a_{3}\right)\right),
\end{aligned}
$$

depending on a parameter $\alpha \in \mathrm{k}$. We did not write the signs explicitly, because they equal the Koszul sign of the permutation of $\left(\Delta, a_{1}, a_{2}, a_{3}\right)$ in the corresponding term. For instance, $\varepsilon$ at the last term in the first line means $\left|a_{1}\right|+\left|a_{2}\right|$. 
The operation $m_{4}$ decomposes into the sum $m_{4}^{\prime}+m_{4}^{\prime \prime}$, where $m_{4}^{\prime}$ is the part that contains $a_{1}, a_{2}, a_{3}, a_{4}$ in this order. The $m_{4}^{\prime}$-part is parametrized by $\alpha \in \mathbb{k}$ as above and another parameter $\beta \in \mathbb{k}$ by

$$
\begin{aligned}
& m_{4}^{\prime}\left(a_{1}, a_{2}, a_{3}, a_{4}\right) \\
& =(1+\beta) \Delta\left(a_{1} a_{2} a_{3} a_{4}\right)+(2 \alpha-\beta)\left(\Delta\left(a_{1}\right) a_{2} a_{3} a_{4}+(-1)^{\varepsilon} a_{1} a_{2} a_{3} \Delta\left(a_{4}\right)\right) \\
& \quad+(4 \alpha-\beta)\left((-1)^{\varepsilon} a_{1} \Delta\left(a_{2}\right) a_{3} a_{4}+(-1)^{\varepsilon} a_{1} a_{2} \Delta\left(a_{3}\right) a_{4}\right) \\
& \quad-(1+\alpha)\left(\Delta\left(a_{1} a_{2} a_{3}\right) a_{4}+(-1)^{\varepsilon} a_{1} \Delta\left(a_{2} a_{3} a_{4}\right)\right) \\
& \quad-\alpha\left(\Delta\left(a_{1} a_{2}\right) a_{3} a_{4}+(-1)^{\varepsilon} a_{1} a_{2} \Delta\left(a_{3} a_{4}\right)\right)+(1-\alpha)(-1)^{\varepsilon} a_{1} \Delta\left(a_{2} a_{3}\right) a_{4} .
\end{aligned}
$$

If the vanishing of $m_{3}$ implies the vanishing of $m_{4}$, then $m_{4}^{\prime}$ must equal the linear combination

$$
\begin{gathered}
A m_{3}\left(a_{1} a_{2}, a_{3}, a_{4}\right)+\operatorname{Bm}_{3}\left(a_{1}, a_{2} a_{3}, a_{4}\right)+C m_{3}\left(a_{1}, a_{2}, a_{3} a_{4}\right) \\
+D a_{1} m_{3}\left(a_{2}, a_{3}, a_{4}\right)+E m_{3}\left(a_{1}, a_{2}, a_{3}\right) a_{4}
\end{gathered}
$$

with some $A, \ldots, E \in \mathbb{k}$. Expanding $m_{3}$ 's in the above display gives the system:

$$
\begin{aligned}
(1+\beta) & =(1+\alpha)(A+B+C), \\
(2 \alpha-\beta) & =-\alpha(B+C+E)=-\alpha(A+B+D), \\
(4 \alpha-\beta) & =(1-\alpha) C-\alpha D-(\alpha-1) E=(1-\alpha) A-\alpha E-(\alpha-1) D, \\
\alpha & =\alpha A+C+E=\alpha C+A+D, \\
(1-\alpha) & =(1-\alpha) B-D-E, \text { and } \\
(\alpha+1) & =A+B-(\alpha+1) E=B+C-(\alpha+1) D .
\end{aligned}
$$

The case $C_{3}=1$ corresponds to $\alpha=0$ by (34). The 2 nd equation of (36) immediately gives that $\beta=0$, so the corresponding $m_{2}$ and $m_{3}$ equal the Börjeson braces $b_{2}^{\Delta}$ and $b_{3}^{\Delta}$ as claimed.

The $C_{3}=-1$ case happens when $\alpha=-2$. One may verify that then the system (36) has the unique solution, namely

$$
\alpha=\beta=-2, A=C=1 / 2, B=0 \text { and } D=E=-3 / 2 .
$$

Therefore, the only way how to express $m_{4}^{\prime}$ as a linear combination (35) is

$$
\begin{aligned}
m_{4}^{\prime}\left(a_{1}, a_{2}, a_{3}, a_{4}\right) \\
=\frac{1}{2}\left(m_{3}\left(a_{1} a_{2}, a_{3}, a_{4}\right)+m_{3}\left(a_{1}, a_{2}, a_{3} a_{4}\right)\right) \\
\quad-\frac{3}{2}\left(a_{1} m_{3}\left(a_{2}, a_{3}, a_{4}\right)+m_{3}\left(a_{1}, a_{2}, a_{3}\right) a_{4}\right),
\end{aligned}
$$

so $m_{3}=0$ does not imply $m_{4}=0$ over $\mathbb{Z}$. This excludes this case.

Remark 4.6 In the $C_{3}=1$ case of the above proof, the solutions of the system (36) form a 2-parametric family depending on $A, B \in \mathbb{k}$ : 


$$
\alpha=\beta=0, C=1-(A+B), D=-A \text { and } E=(A+B)-1 .
$$

The particular solution with $B=1$ and all other parameters trivial gives rise to the 'canonical' recursion

$$
b_{4}^{\Delta}\left(a_{1}, a_{2}, a_{3}, a_{4}\right)=b_{3}^{\Delta}\left(a_{1}, a_{2} a_{3}, a_{4}\right)
$$

that generalizes to all higher Börjeson's braces in the obvious way, proving their hereditarity.

Example 4.7 (Non-hereditary braces) Proposition 4.4 can be used to produce examples of non-hereditary braces. Recall from Example 2.4 that the Börjeson braces are generated by the automorphism $\phi$ with the generating series $\phi(t):=t+t^{2}+t^{3}+$ $t^{4}+\cdots$. Assume that $\mathcal{A}=\left(A, \Delta, m_{2}, m_{3}, \ldots\right)$ is the $A_{\infty}$-algebra given by the automorphism $\tilde{\phi}$ with the generating series of the form

$$
\tilde{\phi}(t):=t+t^{2}+t^{3}+\text { higher order terms. }
$$

Clearly $m_{2}=b_{2}^{\Delta}, m_{3}=b_{3}^{\Delta}$. By Proposition $4.4, \mathcal{A}$ is hereditary if and only if $\phi(t)=\tilde{\phi}(t)$. So choosing e.g. $\tilde{\phi}(t):=t+t^{2}+t^{3}$ produces non-hereditary braces.

Example 4.8 (Super-exotic $A_{\infty}$-braces) We present $A_{\infty}$-braces that are natural in the extended sense of Example 3.7. They are given, for elements $a, a_{1}, a_{2}, \ldots$ of a graded associative algebra $A$ with a differential $\Delta$, by

$$
\begin{aligned}
& s_{1}^{\Delta}(a)= \Delta(a), \\
& s_{2}^{\Delta}\left(a_{1}, a_{2}\right)= \begin{cases}\Delta\left(a_{1} a_{2}\right), & \text { if } \quad\left(\left|a_{1}\right|,\left|a_{2}\right|\right)=(0,0), \\
-\Delta\left(a_{1}\right) a_{2}, & \text { if } \quad\left(\left|a_{1}\right|,\left|a_{2}\right|\right)=(-1,0), \\
-a_{1} \Delta\left(a_{2}\right), & \text { if } \quad\left(\left|a_{1}\right|,\left|a_{2}\right|\right)=(0,-1), \quad \text { and } \\
0, & \text { in the remaining cases, }\end{cases} \\
& s_{3}^{\Delta}\left(a_{1}, a_{2}, a_{3}\right)= \begin{cases}\Delta\left(a_{1} a_{2} a_{3}\right), & \text { if } \quad\left(\left|a_{1}\right|,\left|a_{2}\right|,\left|a_{3}\right|\right)=(0,0,0), \\
a_{1} \Delta\left(a_{2}\right) a_{3} & \text { if } \quad\left(\left|a_{1}\right|,\left|a_{2}\right|,\left|a_{3}\right|\right)=(0,-1,0), \quad \text { and } \\
0 & \text { in the remaining cases, }\end{cases} \\
& s_{k}^{\Delta}\left(a_{1}, \ldots, a_{k}\right)= \begin{cases}\Delta\left(a_{1} \cdots a_{k}\right), & \text { if } \quad\left(\left|a_{1}\right|, \ldots,\left|a_{k}\right|\right)=(0, \ldots, 0), \quad \text { and } \\
0 & \text { in the remaining cases, } k \geq 4 .\end{cases}
\end{aligned}
$$

The above braces are the Börjeson braces of the couple $\left(A^{\prime}, \Delta\right)$, where $A^{\prime}$ is the associative algebra with the same underlying space as $A$ but the multiplication $\cdot$ defined as

$$
a \bullet^{\prime} b:= \begin{cases}a b, & \text { if }(|a|,|b|)=(0,0), \text { and } \\ 0, & \text { in the remaining cases. }\end{cases}
$$


Notice that the above braces are nontrivial even when $\Delta$ is a derivation! For this reason we did not consider this kind of extended naturality.

Commutative associative case. The first part of this section translates to the commutative case in a straightforward manner, so we formulate only the commutative versions of the main theorems. We start with the commutative variant of Theorem 4.1. Recall that $\mathcal{L}_{\Delta}$ denotes the trivial $L_{\infty}$-algebra from Example 2.7.

Theorem 4.9 For each natural $L_{\infty}$-algebra $\mathcal{L}=\left(A, l_{1}, l_{2}, l_{3}, \ldots\right)$ such that

$$
l_{1}=\Delta
$$

there exist a unique natural automorphism $\phi=\left(11, \phi_{2}, \phi_{3}, \ldots\right)$ of the symmetric coalgebra $\mathbb{S}^{c} A$ such that $\mathcal{L}$ equals the twisting of $\mathcal{L}_{\Delta}$ via $\phi$. The $L_{\infty}$-algebra $\mathcal{A}=$ $\left(A, l_{1}, l_{2}, l_{3}, \ldots\right)$ satisfies

$$
l_{2}\left(a_{1}, a_{2}\right)=\Delta\left(a_{1} a_{2}\right)-(-1)^{\left|a_{1}\right|} a_{1} \Delta\left(a_{2}\right)-\Delta\left(a_{1}\right) a_{2}, a_{1}, a_{2} \in A,
$$

if and only if $\phi_{2}$ equals the product of $A$.

Theorem 4.9 combined with the description of natural automorphisms given in Example 3.12 leads to:

Corollary 4.10 Natural $L_{\infty}$-algebras satisfying (37) are parametrized by power series

$$
\phi(t):=t+f_{2} t^{2}+f_{3} t^{3}+f_{4} t^{4}+\cdots \in \mathbb{k}[[t]] .
$$

Natural $L_{\infty}$-algebras satisfying also (38) are parametrized by series (39) with $f_{2}=1$.

The following theorem offers a characterization of Koszul $L_{\infty}$-braces analogous to that of Börjeson $A_{\infty}$-braces given in Theorem 4.3.

Theorem 4.11 The Koszul braces $\left(A, \Delta, \Phi_{2}^{\Delta}, \Phi_{3}^{\Delta}, \Phi_{4}^{\Delta}, \ldots\right)$ are the unique natural $L_{\infty}$-braces defined over $\mathbb{Z}$ such that

(i) $\Phi_{2}^{\Delta}$ measures the deviation of $\Delta$ from being a derivation, i.e.

$$
\Phi_{2}^{\Delta}\left(a_{1}, a_{2}\right)=\Delta\left(a_{1} a_{2}\right)-\Delta\left(a_{1}\right) a_{2}-(-1)^{\left|a_{1}\right|} a_{1} \Delta\left(a_{2}\right), a_{1}, a_{2} \in A,
$$

(ii) the coefficient at $\Delta\left(a_{1} a_{2} a_{3}\right)$ in $\Phi_{3}^{\Delta}\left(a_{1}, a_{2}, a_{3}\right)$ is either +1 or -1 and,

(iii) the hereditarity is satisfied, that is for all $k \geq 1$, that is

$$
\Phi_{k}^{\Delta}=0 \text { implies } \Phi_{k+1}^{\Delta}=0 .
$$

Exercise Explain how to construct non-hereditary $L_{\infty}$-braces.

Acknowledgments I would like to express my thanks to Maria Ronco for Remark 2.9 and Kaj Börjeson for spotting a mistake in Example 1.3. I am also indebted to Olga Kravchenko and the referee for many other useful suggestions. 


\section{References}

1. Akman, F.: On some generalizations of Batalin-Vilkovisky algebras. J. Pure Appl. Algebra 120(2), 105-141 (1997)

2. Akman, F., Ionescu, L.M.: Higher derived brackets and deformation theory. I. J. Homotopy Relat. Struct. 3(1), 385-403 (2008)

3. Alfaro, J., Bering, K., Damgaard, P.H.: Algebra of higher antibrackets. Nuclear Phys. B 478(1-2), 459-503 (1996)

4. Batanin, M.A., Markl, M.: Crossed interval groups and operations on the Hochschild cohomology. Preprint arXiv:0803.2249. March 2008.

5. Bering, K.: Non-commutative Batalin-Vilkovisky algebras, homotopy Lie algebras and the Courant bracket. Comm. Math. Phys. 274(2), 297-341 (2007)

6. Börjeson, K.: $A_{\infty}$-algebras derived from associative algebras with a non-derivation differential. Preprint arXiv:1304.6231, April 2013.

7. Koszul, J.-L.: Crochet de Schouten-Nijenhuis et cohomologie. Astérisque, (Numero Hors Serie), 257271 (1985) (The mathematical heritage of Elie Cartan (Lyon, 1984)).

8. Lada, T., Mark1, M.: Strongly homotopy Lie algebras. Comm. Algebra 23(6), 2147-2161 (1995)

9. Lada, T., Stasheff, J.D.: Introduction to SH Lie algebras for physicists. Internat. J. Theoret. Phys. 32(7), 1087-1103 (1993)

10. Markl, M.: A cohomology theory for $A(m)$-algebras and applications. J. Pure Appl. Algebra 83(2), 141-175 (1992)

11. Markl, M.: Loop homotopy algebras in closed string field theory. Comm. Math. Phys. 221(2), 367-384 (2001)

12. Markl, M.: Operads and PROPs. In: Handbook of Algebra, vol. 5, pp. 87-140. Elsevier/North-Holland, Amsterdam (2008).

13. Markl, M., Shnider, S., Stasheff, J.D.: Operads in algebra, topology and physics. In: Mathematical Surveys and Monographs, vol. 96. American Mathematical Society, Providence, RI (2002).

14. Reutenauer, C.: Theorem of Poincaré-Birkhoff-Witt, logarithm and symmetric group representations of degrees equal to Stirling numbers. In: Combinatoire énumérative (Montreal, Que., 1985/Quebec, Que., 1985). Lecture Notes in Mathematics, vol. 1234, pp 267-284. Springer, Berlin (1986).

15. Stasheff, J.D.: Homotopy associativity of $H$-spaces. I. Trans. Amer. Math. Soc. 108, 275-292 (1963)

16. Stasheff, J.D.: Homotopy associativity of $H$-spaces. II. Trans. Amer. Math. Soc. 108, 293-312 (1963)

17. Voronov, T.: Higher derived brackets and homotopy algebras. J. Pure Appl. Algebra 202(1-3), 133-153 (2005) 